J Proteome Res. 2021 February 05; 20(2): 1229-1242. doi:10.1021/acs.jproteome.0c00604.

\title{
Generation of an Interactome for the Tetratricopeptide Repeat Domain of O-GIcNAc Transferase Indicates a Role for the Enzyme in Intellectual Disability
}

\author{
Hannah M. Stephen ${ }^{1}$, Jeremy L. Praissman ${ }^{1}$, Lance Wells ${ }^{1,}$ \\ ${ }^{1}$ Department of Biochemistry and Molecular Biology, Complex Carbohydrate Research Center, \\ University of Georgia, Athens, Georgia 30605, United States of America
}

\begin{abstract}
The O-GlcNAc transferase (OGT) modifies nuclear and cytoplasmic proteins with $\beta$-N-AcetylGlucosamine (O-GlcNAc). With thousands of O-GlcNAc modified proteins but only one OGT encoded in the mammalian genome, a prevailing question is how OGT selects its substrates. Prior work has indicated that the tetratricopeptide repeat (TPR) domain of OGT is involved in substrate selection. Furthermore, several variants of OGT causal for X-linked intellectual disability (XLID) occur in the TPR domain. Therefore, we adapted the BioID labeling method to identify interactors of a TPR-BirA* fusion protein in HeLa cells. We identified 115 interactors representing known and novel O-GlcNAc modified proteins and OGT interactors (Raw data deposited in MassIVE, Dataset ID MSV000085626). The interactors are enriched in known OGT processes (e.g. chromatin remodeling) as well as processes in which OGT has yet to be implicated (e.g. pre-mRNA processing). Importantly, the identified TPR interactors are linked to several disease states but most notably are enriched in pathologies featuring intellectual disability that may underlie the mechanism by which mutations in OGT lead to XLID. This interactome for the TPR domain of OGT serves as a jumping off point for future research exploring the role of OGT, the TPR domain, and its protein interactors in multiple cellular processes and disease mechanisms, including intellectual disability.
\end{abstract}

\section{Graphical Abstract}

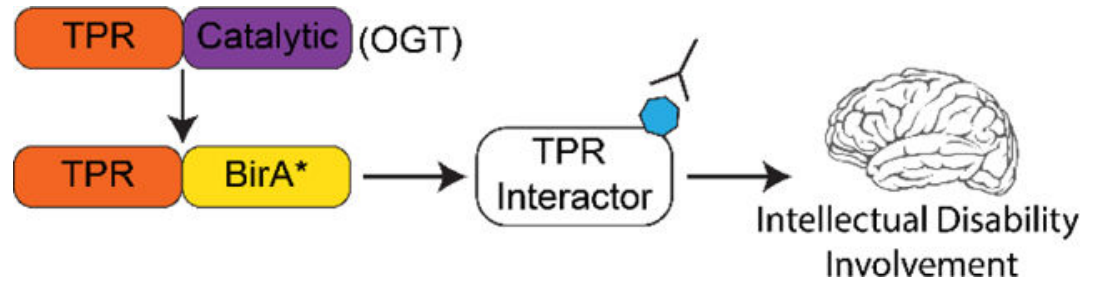

*Correspondence to: 1wells@ccrc.uga.edu.

Author Contributions

H.S. and L.W. conceived and coordinated the study and wrote the manuscript. H.S. performed all experiments and data searches. J.P. performed calculations and statistical analyses on final protein lists and assisted in revision of the manuscript.

Supporting Information

The following supporting information is available free of charge at ACS website http://pubs.acs.org

Raw mass spectrometry data: Deposited in the MassIVE database (https://massive.ucsd.edu/ProteoSAFe/static/massive.jsp, Dataset ID: MSV000085626) 


\section{Keywords}

OGT; TPR; O-GlcNAc; Proximity Proteomics; Mass Spectrometry; Biotin ligase; X-Linked Intellectual Disability

\section{Introduction}

The O-GlcNAc transferase (OGT) is a nucleocytoplasmic glycosyltransferase that modifies substrate proteins with a $\beta-\mathrm{N}$-acetylglucosamine $(\mathrm{O}-\mathrm{GlcNAc})$ on serine and threonine residues. OGT is a unique mammalian glycosyltransferase in that it modifies intracellular proteins outside of the secretory pathway, and the O-GlcNAc modification it creates is non-extended, dynamic, and inducible ${ }^{1-3}$. The O-GlcNAc modification is often compared to phosphorylation, given their similar characteristics and the fact that both occur on thousands of nuclear and cytosolic proteins ${ }^{4-6}$. In fact, OGT and Ser/Thr kinases often compete for the same sites on certain protein substrates ${ }^{4}$ and can regulate each other by posttranslational modification ${ }^{7,8}$. However, unlike protein phosphorylation which is mediated by hundreds of kinases, there is only one gene encoding intracellular O-GlcNAc Transferase in mammals. Thus, not surprisingly, OGT is necessary for the development of mammalian life $^{9}$ and is involved in many intracellular processes including nutrient sensing, transcription, and cellular stress ${ }^{10}$. OGT also has been implicated in many diseases including cancer, Alzheimer's disease, diabetes, and more recently, in X-Linked Intellectual Disability (XLID) as identified originally by our team in collaboration with clinical partners ${ }^{11}$ and further confirmed and expanded on by our group and others ${ }^{12-14}$.

Given the wide diversity of OGT substrates and functions, and the existence of only one mammalian OGT, a prevailing question in the O-GlcNAc field is how OGT selects its substrates. Previous research suggests that the $\mathrm{N}$-terminal tetratricopeptide repeat (TPR) domain of OGT (consisting of 13.5 repeats in the full-length protein), rather than its C-terminal catalytic domain, is responsible for OGT substrate selectivity ${ }^{15-17}$. However, the hypothesis of the TPR domain mediating protein-protein interactions has only been directly tested for a few select proteins ${ }^{18-21}$. A few early attempts to define the full-length OGT-interactome using co-immunoprecipitation have also been made ${ }^{22,23}$. An unbiased approach to identifying proteins that interact specifically with the TPR domain would lend further support to the hypothesis of the TPR domain mediating OGT substrate selectivity, and allow for the identification of new potential substrates and "partner proteins", which interact with the TPR domain of OGT to target it to specific subcellular regions and/or protein complexes.

An additional impetus for TPR interaction studies is the observation that the majority of reported missense mutations in $O G T$ causal for XLID are localized to the TPR domain and do not grossly affect catalytic activity or stability, suggesting a potential protein-protein interaction-based mechanism ${ }^{11,12}$. Therefore, to demonstrate that the TPR domain of OGT is capable of substrate selection, and to capture endogenous OGT TPR interactors including transient interactors, we took advantage of the BioID method, utilizing a fusion protein 
consisting of the full-length OGT TPR domain with a modified biotin ligase in place of the catalytic domain of OGT.

BioID is a well-established proximity proteomic labeling method that utilizes a promiscuous biotin ligase (BirA*) to label nearby proteins with biotin, which allows them to easily be extracted and identified using mass-spectrometry based proteomics ${ }^{24-27}$. Using a TPRBirA* fusion protein in a HeLa cell system (and a eGFP-BirA* fusion protein as a negative control), we have identified over 100 OGT TPR interactors, including both known and novel OGT substrates and interactors. This work strongly suggests that the TPR domain, through protein-protein interactions, plays a major role in OGT substrate selectivity. Exploiting these interactions may allow for fine-tuning of the modification of specific O-GlcNAc modified substrates which has been explored using other techniques ${ }^{28,29}$. This protein set also further supports OGT's role in many cellular processes and reveals potential novel pathways in which O-GlcNAc may play an intricate role. Finally, the interactome is highly enriched in proteins involved in neurological disorders that present with intellectual disability. These proteins represent a set of candidate interactors to explore for future mechanistic studies of the functional role of OGT and the O-GlcNAc modification in XLID.

\section{Experimental Procedures}

\section{Plasmid Constructs}

Plasmids for proximity proteomics were constructed on a CMV promoter with a C-terminal BirA*. Fusion gene construction is as follows. For TPR BirA*: TPR - 3X GGGGS linker BirA* - 2X FLAG. For eGFP-BirA*: eGFP - 3X GGGGS Linker - BirA* - 2XFLAG. The BirA* sequence was obtained from the original paper describing BioID ${ }^{24}$. For TPR-BirA*, residues 1-473 of OGT (consisting of the 13.5 TPR repeats of full-length OGT, Uniprot Accession O15294) were used. Full fusion protein sequences are in Supplementary Table 1.

\section{Cell culture and BiolD}

HeLa cells were grown in DMEM with $10 \%$ FBS on $14.5 \mathrm{~cm}$ plates, passaged for maintenance every 4-6 days (1:4-1:10). For expression of fusion proteins, cells were transfected at $\sim 70 \%$ confluency using lipofectamine 2000 (Invitrogen) according to manufacturer recommended ratios with $60 \mu \mathrm{g}$ plasmid DNA for TPR-BirA* and $6 \mu \mathrm{g}$ plasmid DNA for eGFP-BirA* (eGFP-BirA* expresses at a much higher level than TPR-BirA* - see Fig. 1 C/D). After 24 hours, cell media was replaced with media containing 50uM biotin to induce labeling for 24 hours. Cells were then collected and subjected to nucleocytoplasmic lysis to collect protein. Briefly, cells were lysed in hypotonic buffer A (10mM Tris- $\mathrm{HCl}$ pH 5.5, 500uM DTT, 500uM EDTA, protease and phosphatase inhibitor cocktails (Sigma)) for 10 minutes, vortexed twice for 30 seconds, then lysed in hypertonic buffer B (10mM Tris- $\mathrm{HCl} \mathrm{pH} \mathrm{7.5,} \mathrm{800mM} \mathrm{NaCl,} \mathrm{protease} \mathrm{and} \mathrm{phosphatase} \mathrm{inhibitors,} \mathrm{and} \mathrm{universal} \mathrm{nuclease}$ (ThermoFisher Scientific)) for 5 minutes, followed by 30 seconds of high-speed vortexing and an additional 5 minute incubation. Membranes were pelleted by centrifugation, and the supernatant containing cytosolic and nuclear proteins used in further analysis. Three biological replicates were performed, each consisting of one TPR-BirA* and one eGFPBirA* sample, with TPR-BirA* representing the experimental condition and eGFP-BirA* 
representing the negative control. For supplementary figure 1, HeLa cells were transfected as described and lysed in RIPA buffer.

\section{Quantification of FLAG-tagged proteins}

FLAG-tagged fusion proteins were quantified from HeLa cell lysate using a FLAG ELISA (Cayman Chemical) according to the manufacturer's protocol.

\section{Identification of biotinylated proteins}

Biotinylated proteins were purified from cellular lysate using an anti-biotin immunoprecipitation protocol as previously described ${ }^{30} .25 \%$ of eluate was reserved for anti-biotin western blot, then remaining biotinylated proteins were then run out on a $10 \%$ SDS-PAGE gel using the Bio-Rad Mini-PROTEAN gel system. The gels were not stained. Each lane was cut into four equal fractions based on molecular weight markers, then extracted, reduced, alkylated, and digested with trypsin as previously described ${ }^{30}$. Dried peptides were resuspended in $40 \mu \mathrm{L}$ sample buffer $(10 \%$ Buffer B $(80 \%$ acetonitrile, $0.1 \%$ formic acid), $90 \%$ Buffer A ( $0.1 \%$ formic acid), and $15 \mathrm{uL}$ of this was injected for each instrument run. LC-MS/MS was performed on an Orbitrap Fusion Tribrid mass spectrometer (ThermoFisher Scientific) equipped with an Ultimate 3000 RSLCnano HPLC system (Thermofisher Scientific). Peptides were separated on an Acclaim ${ }^{\mathrm{TM}}$ PepMap ${ }^{\mathrm{TM}}$ RSLC C18 column $(75 \mu \mathrm{m} \mathrm{ID} \times 15 \mathrm{~cm} ; 2 \mu \mathrm{m}$ particle size $)$ at a flow rate of $0.200 \mu \mathrm{L} / \mathrm{min}$ over a 150min linear gradient of $1-99 \%$ Buffer B with a total run time of $180 \mathrm{~min}$. Precursor scans were collected using the Orbitrap mass analyzer with a scan range of $300-2000 \mathrm{~m} / \mathrm{z}$ and mass resolution of 60,000. Most intense ions were fragmented using 38\% CID collision energy and detected in the Ion Trap with 1 microscan and dynamic exclusion for 15 seconds after one occurrence. Samples were run in randomized pairs within a replicate, with each pair consisting of a corresponding gel fraction in eGFP-BirA*, run first, then TPR-BirA*, run second, with one 90 min wash in between each sample and two 90 minute washes in between pairs (20uL $10 \%$ Buffer B injection for washes). The raw data for all 24 LC-MS/MS analyses (12 control and 12 experimental) has been deposited to the MassIVE database (https://massive.ucsd.edu/ProteoSAFe/static/massive.jsp, Dataset ID: MSV000085626).

\section{Data analysis}

Raw files were searched with no prior peaklist selection by both ProteinMetrics Inc. Byonic (v3.8.13) and ThermoFisher Scientific Proteome Discoverer - SequestHT (2.2.0.338). The proteomic database consisted of all SwissProt annotated human protein sequences (obtained 09-2019-20,434 sequences), plus a list of common contaminants (trypsin, keratins, and serum albumins - 179 sequences, available in Supplementary Table 1) and the sequences for eGFP and BirA*. A concatenated database was generated for FDR calculations by including reversed protein sequences for all proteins in the database, creating a final database of 41230 sequences, all of which were searched unbiasedly. Tryptic cleavage was fully specific at Arg and Lys, with two missed cleavages allowed. For both search engines, precursor mass tolerance was 20ppm and fragment mass tolerance was 0.5Da. Carbamidomethylation on Cys was set as a fixed modification, and variable modifications were allowed: Oxidation 
of Met, HexNAc on Ser/Thr, and Biotin on Lys. Full peptide lists were exported from PMI-Byonic and SequestHT with no score cuts.

Peptide lists from each sample (four fractions each) were then combined using ProValt ${ }^{31}$. Peptide cuts were made based on peptide FDR, set at $2 \%$ for the eGFP-BirA* and $1 \%$ for TPR-BirA* - with the lower stringency of filtering for eGFP-BirA* samples selected to help decrease the incidence of potential false positives in the final protein set. Protein lists were generated by removing all peptides not identified by both Byonic and Proteome Discoverer (peptides required to have a positive Byonic Score and Sequest Xcorr), and by removing any proteins that were identified by fewer than two peptides. Only proteins identified as the top protein from among isoforms were kept in the final protein list. Spectral counts were converted to normalized spectral abundance factors (NSAFs) for analysis ${ }^{32}$. The normal logarithm (ln) of NSAF values was computed to create a more Gaussian/normal distribution, and the Student's T-Test was utilized to determine the significance of the difference in abundance between experimental and negative control conditions for each potential interacting protein. Protein IDs with a pvalue of 0.05 or less and with at least a 3 -fold higher NSAF were kept in the final TPR-BirA* interactome.

Further quantification was carried out by reconstructed ion chromatogram (RIC) analysis of peptide intensity performed in Xcalibur Qual Browser (v2.0.3.2). Peptides for analysis were selected based on their appearance in all three replicates in both TPR-BirA* and eGFP-BirA*. Peptide intensity was examined for all gel fractions in which that peptide occurred (For HCF1: fractions 1 and 2; for OGT: fraction 1 [rep 3] and fraction 2 [reps 1,2]; for KNL1: fraction 1). Time ranges for intensity analysis were selected to be the same in TPR-BirA* and eGFP-BirA*. The time range was selected based on the area of overlap between the corresponding peaks, or, in the case of peaks with a slight time offset leading to no overlap, the time range was selected so that the time was evenly split between the two peaks. Peptide intensity was determined by the normalization level (NL) of the monoisotopic peak. Peptide identity was validated by recorded retention time in ProteomeDiscoverer 2.2.

\section{Western Blots and Antibodies}

SDS-PAGE gels (4-15\%) were run using the BioRad Mini-PROTEAN gel system. Gels were transferred onto Immobilin-FL PVDF membranes (Sigma) using the BioRad TransBlot SD Semi-Dry Transfer Cell. Membranes were blocked in 1\% cold water fish skin gelatin (Sigma), then incubated with primary antibody at these ratios: anti-FLAG (1:2500, Sigma F3165), anti-biotin (1:1000, Jackson 200-002-211), anti-OGT (1:1000, Santa Cruz sc-74546), anti- $\beta$-actin (WB 1:2000, Cell Signaling 4970) Histone H3 (1:1000, Cell Signaling 14269), anti-GAPDH (WB, Cell Signaling 2118). Secondary antibodies were LiCor IRDye: 680RD donkey-anti mouse 680 (1:10000), 800CW Goat anti-rabbit (1:20000). Three washes in TBST ( $0.1 \%$ tween) were performed after each antibody incubation. All Western blots were imaged on a LiCor Odyssey Clx system. Densitometric measurements were made using Image Studio Lite v5.2 


\section{Localization studies (Nucleocytoplasmic Fractionation)}

Protein localization was determined using UniProt ${ }^{33}$. Nuclear and cytoplasmic fractions from HeLa cells were obtained using subcellular fractionation as previously described ${ }^{34}$ and analyzed via western blot as above.

\section{Pathway Analysis}

Gene ontology analysis was performed using The Gene Ontology Resource (geneontology.org) ${ }^{35,36}$. All GO lists were filtered at pvalue less than 0.01, FDR of less than 0.01 , and a minimum of 5 -fold enrichment over expected number of proteins found in that category in a random protein dataset. Biological process and molecular function analysis were performed using the GO Ontology Database Released 2019-12-09. ReViGo ${ }^{37}$ was used to generate condensed lists of GO terms and $\mathrm{CirGo}^{38}$ to generate plots from the condensed data. PANTHER pathway analysis was performed using PANTHER version 15 released 2020-02-14. Reactome data was also obtained from The Gene Ontology Resource, using Reactome version 65 released 2019-12-22, and parsed at FDR less than 5E-9. Condensed GO term lists and the full reactome pathway list are available in Supplementary Table 5.

Disease association for proteins was identified using the OMIM catalog 39 . Disorders were categorized manually, where "Intellectual Disability" refers to any disorder with the symptom intellectual disability (or several other related terms), "Immunodeficiency" refers to disorders causing immunodeficiency, "Malignancy" refers to any of several cancers, "Congenital, other" refers to congenital disorders not featuring intellectual disability, "Neurological, other" refers to non-congenital neurological disorders, and "Hormone" refers to disorders of the endocrine system.

\section{Results}

\section{Defining the OGT TPR Interactome in HeLa Cells}

To identify OGT TPR interactors, we utilized a fusion protein strategy using promiscuous biotin ligase BirA*. We generated a fusion protein TPR-BirA*, essentially replacing the catalytic domain of OGT with BirA*, and also created an eGFP-BirA* fusion protein to serve as a negative control for nonspecific protein interactions or promiscuous labeling (Supplementary Table 1). Each fusion protein was transiently overexpressed in HeLa cells and induced with biotin for 24 hours for labeling of proximal proteins (Fig. 1A). A comparison of the expression level of the TPR-BirA* fusion protein and endogenous OGT can be found in Supplementary Figure 1. Note that for transfections, 10x more TPR-BirA* plasmid was used than eGFP-BirA*, due to eGFP-BirA* expressing at a much higher level than TPR-BirA* (Fig. 1C/D). Following labeling, we isolated biotinylated proteins with a biotin immunopurification (Fig. 1E). Even though there is notable biotin labeling in both TPR-BirA* and eGFP-BirA*, we observe a difference in the band patterns, indicating a change in the specificity of biotinylation between eGFP-BirA* and TPR-BirA*. A sectioned SDS-PAGE gel was subjected to in-gel digestion and the resulting peptides separated by nanoflow reverse-phase liquid chromatography in-line to a tribrid mass spectrometer for protein identification (see methods). This entire procedure (transfection to LC-MS/MS 
analyses) was carried out in 3 independent biological replicates for both TPR-BirA* and eGFP-BirA*.

For analysis of the mass spectrometry data, we opted for a multi-algorithm search to increase the confidence of our protein IDs (Fig. 1B). Raw mass spectrometry data was searched using both PMI-Byonic and Sequest HT (through ProteomeDiscoverer 2.2) against the human database (Swissprot 09/2019), and only peptides identified by both algorithms were used to generate the final protein set. The negative control (eGFP-BirA*) protein set was searched at a looser peptide FDR $(2 \%)$ than the TPR protein set $(1 \%)$ to reduce the likelihood of proteins being kept in the final TPR interactors list due to barely being over the FDR threshold in the control condition. To generate proteins for a final TPR interactors list, we combined uniquely identified proteins in the TPR-BirA* analyses with those that were enriched in the TPR-BirA* protein lists as compared to the eGFP-BirA* (negative control) protein lists. This enrichment was required to be significant according to the Student's t-test with the cut-off for significance being a p-value of 0.05 , and we also required the average $\mathrm{NSAF}^{32}$ to be at least 3 times higher in the TPR-BirA* condition compared to the eGFP-BirA* condition. These proteins represent a stringent list of 115 OGT TPR interactors (Table 1, Supplementary Table 4).

46 of the proteins (indicated by an italicized protein name in Table 1) had already been identified as O-GlcNAc modified by previous -omic datasets ${ }^{40,41}$. This result suggests that the TPR domain alone can select OGT substrate proteins without the presence of the catalytic domain. In addition, 8 (indicated by an italicized and bold protein name in Table 1) of the identified proteins had previously been demonstrated to specifically interact with OGT, where "interaction" here is defined as a one or two directional coimmunoprecipitation ${ }^{19-21,42-45}$. Together, these factors lend confidence to the novel protein IDs in the dataset.

It is noteworthy that OGT itself is identified in the screen. Although many peptides attributed to OGT are due to the overexpression of the TPR-BirA* fusion protein, several high-confidence peptides in the catalytic domain of OGT were also identified. This suggests that endogenous OGT, which normally exists as a dimer ${ }^{16}$, did complex with the fusion TPR protein. Several well-studied OGT interactors were also identified, including $\mathrm{HCF}^{21}$, $\mathrm{mSin} 3 \mathrm{a}^{19}$, and Tet $2^{20}$. We also identify the O-GlcNAc hydrolase OGA, which OGT is known to regulate both pre- and post-translationally ${ }^{11,46}$.

Many of the interactors identified here are members of protein complexes which may imply that OGT does not directly interact with all of the proteins in the complex. One such complex is the HIRA protein complex, previously shown to interact with $\mathrm{OGT}^{42}$. We identified all three members of this complex (HIRA, UBN1, and CABIN). We also identified a novel TPR interaction with the SWI/SNF complex. Six members of the SWI/SNF complex were identified (SMRC2, SMRC1, ACL6A, SMCA4, ARI1A, ARI1B). SWI/SNF proteins, like OGT ${ }^{19,20,47-52}$, function in chromatin remodeling ${ }^{53}$, but OGT has, to our knowledge, never been shown to interact with these proteins. In addition to many protein interactors involved in known OGT functions, we also identified proteins with roles in RNA processing, an area of cellular biology for which limited research exists on the role of OGT and the 
O-GlcNAc modification. These interactors include proteins with known and putative roles in pre-mRNA splicing (SF3A1, PCF11, PRC2A, PR40A), polyadenylation (CPSF1), and RNA binding (ZN106, TNR6B, RBM33, RBM25, RBM26).

\section{Validation of Proteins Identified in both TPR-BirA* and eGFP-BirA*}

72 of the 115 OGT TPR interactors were only observed in the TPR interactome. Several protein IDs (43 - indicated by a bold Uniprot accession in Table 1) were identified in both the TPR-BirA* and eGFP-BirA* samples, but were significantly enriched in TPR-BirA* at the level of average NSAF (Student's t-test $p$ value $<0.05$, fold enrichment of average NSAF >3) (Fig. 2A). To further confirm the validity of the inclusion of these proteins in the final dataset, we examined MS1 reconstructed ion chromatograms for peptides identified in both TPR-BirA* and eGFP-BirA*. OGT itself was identified in both, although it is highly enriched in the TPR-BirA*, likely in part due to the overexpression of the TPR-BirA* fusion protein. To confirm that endogenous OGT labeling is enriched in the TPR-BirA* sample, we compared the intensity of a catalytic domain peptide between the TPR-BirA* samples and the eGFP-BirA* samples. The average intensity of this peptide in the TPR-BirA* samples is 9.1 (standard deviation of 2.3) times higher than in the eGFP-BirA* samples, supporting specific interaction of TPR-BirA* with full-length endogenous OGT (average mass error 2.17ppm) (Fig. 3A).

To further confirm the enrichment of relevant OGT interactors in TPR-BirA*, we next examined the intensity of peptides from HCF-1, a well-studied OGT interactor with roles in intellectual disability ${ }^{21,54}$. Average intensity for an HCF-1 peptide are 36.4-fold (standard deviation of 24.4) higher in TPR-BirA* than eGFP-BirA* (average mass error 0.749ppm) (Fig. 3B). Finally, we confirmed a fold increase in peptide intensity for the protein closest to our fold enrichment cutoff, kinetochore scaffold 1 (KNL1) (Fig. 3C). The peptide shown from KNL1 has an average intensity in TPR-BirA* that is 3.93 (standard deviation of 1.93) higher than in eGFP-BirA* (average mass error 2.12ppm). Taken together, these results indicate that although some proteins were identified in both the experimental and negative control conditions, their inclusion in the final protein interactor list due to higher enrichment is supported by the raw mass spectrometry data.

\section{Subcellular Localization of TPR-BirA* Interactors}

OGT localizes primarily to the nucleus, but also to the cytoplasm, in the mammalian cell $^{55}$. To confirm that the TPR-BirA* fusion protein also localized to both compartments, we examined the subcellular localization of OGT and our fusion proteins. By subcellular fractionation (Fig. 4A/B), endogenous OGT localizes primarily to the nucleus with some expression in the cytoplasm, as expected ${ }^{55}$. In contrast, the TPR-BirA* fusion protein localizes more highly to the cytoplasm, although it is also present in the nucleus. This result is unsurprising as previous research has shown that overexpressed OGT localizes more highly to the cytoplasm than native OGT ${ }^{55}$. eGFP-BirA* also localizes to both the nucleus and cytoplasm. The subcellular localization profiles of TPR-BirA* and eGFP-BirA* are very similar, supporting the use of eGFP-BirA* as a sufficient negative control for nonspecific labeling by BirA* in both the nuclear and cytosolic compartments. 
We expected most TPR-BirA* interactors to be primarily nuclear, as most recorded OGT interactors are as well ${ }^{10}$. Analysis of the subcellular localization of identified TPR-BirA* interactors supports this (Visualized in an UpsetR plot Fig. 4C, and in a pie chart in Fig. 4D). 67 of the 115 identified interactors are exclusively nuclear, with an additional 23 occurring in both the nucleus and the cytoplasm. Several interactors localize specifically to the nuclear pore. Only four proteins exclusively localize to the cytoplasm, all of which are novel OGT-TPR interactors (RGPD5, WNK1, TNR6B, and ANKH1). ${ }^{10}$

\section{Ontology Analyses of the OGT TPR-Interactome}

To further understand the enrichment of various processes in our OGT TPR interactome, we performed several different Gene Ontology (GO) analyses using the Gene Ontology Resource (GeneOntology.org) (Fig. 5). Performance of a PANTHER Overrepresentation Test for biological processes in our interactome further confirms OGT-TPR interactors having frequent roles in transcriptional and chromatin regulation (Fig. 5A). General chromatin organization is a strongly enriched category, along with the related peptidyllysine modification (indicative of histone modification). Of note is the enrichment of OGT-TPR interactors specifically involved in gene silencing. OGT is a Polycomb Group Protein (sxc in Drosophila melanogaster), which is responsible for the silencing of Hox genes during developmental patterning 47,48 . The TPR interactors identified here further corroborate OGT's previously-identified tendency toward roles in gene silencing and may reveal further avenues by which OGT regulates gene silencing ${ }^{19}$. Of additional note is the enrichment of interactors involved in the regulation of cellular response to heat, since previous work has demonstrated a role for OGT in coping with cellular heat shock ${ }^{56}$; however, limited work has been published exploring the specific OGT interactions that help it to perform this function. Finally, the enrichment of proteins involved in rhythmic process and circadian rhythm aligns with previous research demonstrating that OGT is involved in circadian rhythm regulation ${ }^{57}$. To confirm these enriched processes, we also performed a PANTHER Overrepresentation Test for molecular function pathways in our OGT TPR interactome (Fig. 5B). Many molecular functions corroborate our findings of enriched biological processes, including consistent high enrichment in chromatin and transcriptional regulation. It is interesting to note that RNA Pol II transcription factor binding in particular is an enriched molecular function, since OGT is known to interact with and regulate RNA Pol II-mediated transcription ${ }^{18,58,59}$. Also enriched are processes specifically relating to histone modification, further confirming the enrichment of peptidyllysine modification of histones as identified in biological process enrichment and consistent with the O-GlcNAc modification being part of the histone code ${ }^{52}$. Finally, enrichment of nuclear pore components and nuclear localization sequence binding confirms the longstanding role for OGT in nuclear pore structure and/or regulation ${ }^{60}$.

We further examined enriched Reactome pathways (Table 2) among TPR interactors, specifically the mostly highly enriched pathways at an FDR of less than 5E-9. This pathway analysis validates our previous GO analyses that reveal roles for OGT in chromatin regulation, transcriptional regulation, and nuclear pore processes. Reactome pathway enrichment also uniquely reveals several roles for OGT TPR interactors in viral infection, nuclear import, and processing. OGT has been demonstrated to play a role in 
a limited number of specific viral infections ${ }^{46,61}$ but these enriched pathways point to a potentially broader role for OGT and its interactors more generally in viral infection. Interactors are also enriched in the reactome pathway "regulation of glucokinase by glucokinase regulatory protein". OGT has already been shown to regulate glucokinase ${ }^{62}$ as well as other proteins involved in glucose metabolism including phosphofructokinase $1^{63}$. Panther pathway enrichment analysis (Fig. 5C) reveals OGT-TPR interactor involvement in basic leucine-zipped transcription factor mediated transcriptional regulation, the Wnt signaling pathway, and Huntington disease related processes. The interplay with basic leucine-zipped transcription factors points to another potential avenue for OGT's regulation of transcription. Furthermore, OGT has already been shown to interface with the Wnt pathway by modulating $\beta$-Catenin stability ${ }^{64}$. The interactors identified here involved in this pathway may point to other mechanisms by which OGT modulates Wnt signaling.

\section{Pathophysiology Analyses of the OGT TPR-Interactome and Orthogonal validation of XLID- related Interactors}

The identification of Huntington's disease (Fig. 5C) as an enriched disease process among the TPR interactors prompted us to examine whether identified TPR interactors are involved in other disease processes. Unsurprisingly, as determined using the OMIM catalogue, many TPR interactors are involved in disease processes with which OGT is already associated, including malignancy ${ }^{65}$ and neurological ${ }^{66}$ disorders (Fig. 6A/B).

Strikingly, of the 44 TPR interactors involved in an OMIM-classified phenotype, 24 are linked to disorders which feature intellectual disability. Three interactors are linked to two different intellectual disability-related disorders each, bringing the total count of intellectual disability disorders associated to OGT-TPR interactors to 27 (Fig 6A/B). 11-1411,12,67 Therefore, these 24 protein interactors are of significant interest in the search for a mechanism underlying the OGT XLID phenotype.

\section{Discussion}

One of the prevailing mysteries in the O-GlcNAc field is how the O-GlcNAc Transferase (OGT) enzyme is able to select from among thousands of possible substrates given that there is only one gene encoding the protein in the mammalian cell. A prevailing hypothesis in the field is that post-translational modification of OGT and protein-protein interactors are responsible for OGT substrate selection. This mechanism may be similar to RNA PolII that transcribes all protein-coding genes but is exquisitely regulated by protein-protein associations including the basal transcriptional machinery and transcription factors ${ }^{68}$. The role of the TPR domain of OGT in promoting highly specific substrate selection has been demonstrated in part in structural biology studies ${ }^{16,17,69,70}$, but has only been explored for a limited number of specific substrates. Here, we demonstrated that the TPR domain of OGT is capable of interacting with substrate proteins even without the presence of the catalytic domain, and using the BioID technique we have identified 115 TPR interactors, representing both known OGT interactors and novel OGT-TPR interactors.

OGT is known to localize to the nucleus and the cytoplasm, but usually primarily resides in the nucleus ${ }^{55}$. The fact that most interactors found here are exclusively localized to 
the nucleus despite the TPR-BirA* fusion protein being localized more dominantly to the cytoplasm is an intriguing observation, suggesting that OGT more strongly and/or frequently interacts with nuclear proteins regardless of localization. It is important to note that the nature of the TPR-BirA* as a truncation of OGT and an overexpressed fusion protein opens the possibility for false-negative or false-positive protein IDs. While we have attempted to thoroughly mitigate the occurrence of false positives through stringent statistical analyses and the use of a negative control (eGFP-BirA*), future work to biochemically confirm each interaction with the full-length OGT is necessary. This will also allow for the determination of the degree to which given interactions are transient or stable, under what conditions they occur, and what functional roles they play in the cell.

The identified interactors likely fall into several different classes. Many of the identified proteins are O-GlcNAc modified, and these may be the effector substrates by which OGT modulates cellular status. Another group, not mutually exclusive to the first, may represent partner proteins; that is, proteins that interact with the TPR domain of OGT to target it to specific substrates or intracellular regions, thus affecting the substrates OGT can access. These proteins may or may not themselves be functionally O-GlcNAc modified. As an example, Tet2 binds to OGT and directs it to histones, but the O-GlcNAc modification on Tet2 has no observed effect on its function ${ }^{20}$. Finally, it is likely that some of the identified interactors do not directly interact with OGT but rather are members of a complex, a subset of which interact directly with OGT. The Swi/Snf complex is an example of this - we have identified 6 of at least 20 possible subunits in our TPR-BirA* interaction list. It is likely that the TPR domain does not directly interact with all 6 identified components and instead interacts with a subset, but additional complex members are labeled due to the diffusion of the reactive biotin intermediate released by the BirA* protein. This would also explain why we fail to identify the full complex; additional members of the complex may be too distant in space to be biotin labeled. Further work is required to identify direct versus indirect interactors.

In general, the TPR interactors identified here corroborate OGT's role as a high-level regulator of cellular function as identified in other studies ${ }^{19,47,48}$. OGT has previously been characterized as a "rheostat" rather than a switch ${ }^{10}$, and the data here supports this notion. Most of the TPR interactors we identified are "modulators" themselves, e.g. they are not enzymes with a direct effect on a given substrate, but rather affect cellular physiology at a global level by modulating transcription, protein stability, or transport. This gives a perspective of OGT as a modulator of the modulators; that is, OGT regulates cellular function by making many subtle changes in global regulators, adding up to a more significant functional outcome. One such global regulation avenue is chromatin remodeling, which is a previously known function of OGT and a function in which many of our TPR interactors are involved. While our data does not determine how OGT's interaction with these chromatin remodelers affects their function, many of our identified interactors are involved in lysine modification of histones, pointing to a potential avenue for OGT's regulation of chromatin remodeling. Indeed, OGT has already been noted to interact with histone modifying enzymes including HDACs ${ }^{19}$. We are unsure why we did not identify any HDACs in our screen - it is possible that they interact with OGT as a part of a protein complex but at a distance outside of the BirA* biotin labeling radius. 
We have also identified interactors involved in biological processes in which OGT has yet to be implicated, most noteworthy in RNA processing and transport. Interestingly, OGA, which removes the O-GlcNAc modification, has previously been shown to localize to the nucleolus ${ }^{71}$, indicating the presence and possible role of O-GlcNAc modified proteins in this subcellular structure involved in RNA processing. Future work will be necessary to determine the specific role OGT plays in these processes.

Finally, we have identified many OGT-TPR interactors that are involved in disease. The TPR interactors we present here may represent avenues for future research uncovering mechanistic proteins underlying OGT's role in various disease states. Of current high interest is the mechanism underlying $O G T$ mutations leading to X-Linked Intellectual Disability (XLID) ${ }^{11,13,72}$. One prominent hypothesis that we have previously suggested ${ }^{11,12,67}$ is that mutations in the TPR domain disrupt OGT protein interactions, leading to downstream developmental effects that lead to the XLID phenotype. Here, we have identified 24 OGT TPR interactors directly involved in disorders with intellectual disability. While it is possible that a novel interactor or set of interactors underlies the OGTXLID mechanism, these interactors represent a strong set of candidate interactors that may contribute to the phenotype. The high number of interactors with connections to intellectual disability may also indicate that there may be a more global interruption in protein-protein interactions caused by XLID variants in OGT. Rather than one specific interactor failing to interact with OGT and leading to XLID, there may be a more subtle reduction in interaction with many proteins, leading to global neurodevelopmental abnormalities.

Our lab is currently undertaking BioID and immunoprecipitation studies to identify any perturbations in the OGT interactome in neural lines harboring XLID-linked OGT variants. The BioID method described here will be a valuable tool to identify potentially tissue- $/$ cell type-specific TPR interactors that fail to interact with XLID-associated OGT variants. In a more directed approach, intellectual disability-related interactors identified here are being screened for protein interaction with XLID-linked OGT variants to determine if they may represent protein interactors underlying the XLID-OGT phenotype. Thus, the work presented here lays a groundwork for additional studies to understand OGT substrate selectivity and the role of OGT and the O-GlcNAc modification in a plethora of biological processes and human pathophysiology including XLID.

\section{Supplementary Material}

Refer to Web version on PubMed Central for supplementary material.

\section{Acknowledgments}

This article is dedicated to the memory of our beloved colleague Dr. Brent Weatherly, who developed the proteomic analysis workflows used in this manuscript. We thank Dr. Kelley Moremen for technical advice and plasmid constructs. This work was supported by a grant from the W.M. Keck foundation (L.W. Co-PI); an NICHD National Institutes of Health (NIH) grant R21HD097652 (L.W); and an NICHD Grant F30 HD098828 (H.S.). The content is solely the responsibility of the authors and does not necessarily represent the official views of the National Institutes of Health. 


\section{References}

1. Hart GW Dynamic O-Linked Glycosylation of Nuclear and Cytoskeletal Proteins. Annu. Rev. Biochem 1997, 66, 315-335. [PubMed: 9242909]

2. Holt GD \& Hart GW The subcellular distribution of terminal N-acetylglucosamine moieties. Localization of a novel protein-saccharidie linkage, O-linked GlcNAc. J. Biol. Chem 1986, 261, 8049-8057. [PubMed: 3086323]

3. Walgren JLE, Vincent TS, Schey KL \& Buse MG High glucose and insulin promote O-GlcNAc modification of proteins, including a-tubulin. Am. J. Physiol. - Endocrinol. Metab 2003, 284, 424 434.

4. Wang Z, Pandey A \& Hart GW Dynamic interplay between O-linked N-acetylglucosaminylation and glycogen synthase kinase-3-dependent phosphorylation. Mol. Cell. Proteomics 2007, 6, 13651379. [PubMed: 17507370]

5. Wang Z, Gucek M \& Hart GW Cross-talk between GlcNAcylation and phosphorylation: sitespecific phosphorylation dynamics in response to globally elevated O-GlcNAc. Proc. Natl. Acad. Sci. U. S. A 2008, 105, 13793-8. [PubMed: 18779572]

6. Wang Z, Udeshi ND, Slawson C, Compton PD, Cheung WD, Shabanowitz J, Hunt DF \& Hart GW Extensive Crosstalk Between O-GlcNAcylation and Phosphorylation Regulates Cytokinesis. Sci Signal 2010, 3, 1-22.

7. Li Z, Li X, Nai S, Geng Q, Liao J, Xu X \& Li J Checkpoint kinase 1-induced phosphorylation of O-linked $\beta-\mathrm{N}$-acetylglucosamine transferase regulates the intermediate filament network during cytokinesis. J. Biol. Chem 2017, 292, jbc.M117.811646.

8. Tarrant MK, Rho H, Xie Z, Jiang YL, Gross C, Culhane JC, Yan G, Qian J, Ichikawa Y, Matsuoka T, Zachara N, Etzkorn FA, Hart GW, Jeong JS, Zhu H \& Cole PA Regulation of CK2 by Phosphorylation and O-GlcNAcylation Revealed by Semisynthesis. Nat. Chem. Biol 2012, 8, 262-269. [PubMed: 22267120]

9. Shafi R, Iyer SPN, Ellies LG, O’Donnell N, Marek KW, Chui D, Hart GW \& Marth JD The O-GlcNAc transferase gene resides on the $\mathrm{X}$ chromosome and is essential for embryonic stem cell viability and mouse ontogeny. Proc. Natl. Acad. Sci 2000, 97, 5735-5739. [PubMed: 10801981]

10. Hart GW Nutrient regulation of signaling and transcription. J. Biol. Chem 2019, 294, 2211-2231. [PubMed: 30626734]

11. Vaidyanathan K, Niranjan T, Selvan N, Teo CF, May M, Patel S, Weatherly B, Skinner C, Opitz J, Carey J, Viskochil D, Gecz J, Shaw M, Peng Y, Alexov E, Wang T, Schwartz C \& Wells L Identification and Characterization of a Missense Mutation in the O-GlcNAc Transferase Gene that Segregates with X-Linked Intellectual Disability. J. Biol. Chem 2017, 292, jbc.M116.771030.

12. Selvan N, George S, Serajee FJ, Shaw M, Hobson L, Kalscheuer V, Prasad N, Levy SE, Taylor J, Aftimos S, Schwartz CE, Huq AM, Gecz J \& Wells L O-GlcNAc transferase missense mutations linked to X-linked intellectual disability deregulate genes involved in cell fate determination and signaling. J. Biol. Chem 2018, 293, 10810-10824. [PubMed: 29769320]

13. Pravata VM, Gundogdu M, Bartual SG, Ferenbach AT \& Wojcik MH A missense mutation in the catalytic domain of O-GlcNAc transferase links perturbations in protein O -GlcNAcylation to X-linked intellectual disability. 2019, 1-11. doi:10.1002/1873-3468.13640

14. Pravata VM, Muha V, 1+ G, Ferenbach AT, Kakade P, Vandadi V, Wilmes AC, Borodkin VS, Joss S, Stavridis MP \& Van Aalten DMF Catalytic deficiency of O-GlcNAc transferase leads to X-linked intellectual disability. 2019, 116, 14961-14970.

15. Iyer SPN \& Hart GW Roles of the Tetratricopeptide Repeat Domain in O-GlcNAc Transferase Targeting and Protein Substrate Specificity. J. Biol. Chem 2003, 278, 24608-24616. [PubMed: 12724313]

16. Jinek M, Rehwinkel J, Lazarus BD, Izaurralde E, Hanover JA \& Conti E The superhelical TPR-repeat domain of O-linked GlcNAc transferase exhibits structural similarities to importin alpha. Nat Struct Mol Biol 2004, 11, 1001-1007. [PubMed: 15361863]

17. Levine Z, Fan C, Melicher MS, Orman M, Benjamin T \& Walker S O-GlcNAc transferase recognizes protein substrates using an asparagine ladder in the TPR superhelix. J. Am. Chem. Soc 2018, jacs.7b13546. doi:10.1021/jacs.7b13546 
18. Comer FI \& Hart GW Reciprocity between O-GlcNAc and O-phosphate on the carboxyl terminal domain of RNA polymerase II. Biochemistry 2001, 40, 7845-7852. [PubMed: 11425311]

19. Yang X, Zhang F \& Kudlow JE Recruitment of O -GlcNAc Transferase to Promoters by Corepressor mSin3A : Coupling Protein O -GlcNAcylation to Transcriptional Repression. Cell 2002, 110, 69-80. [PubMed: 12150998]

20. Chen Q, Chen Y, Bian C, Fujiki R \& Xiaochun Y Tet2 promotes histone O-GlcNAcylation during gene transcription. Nature 2013, 493, 561-564. [PubMed: 23222540]

21. Capotosti F, Guernier S, Lammers F, Waridel P, Cai Y, Jin J, Conaway JW, Conaway RC \& Herr W O-GlcNAc transferase catalyzes site-specific proteolysis of HCF-1. Cell 2011, 144, 376-388. [PubMed: 21295698]

22. Deng RP, He X, Guo SJ, Liu WF, Tao Y \& Tao SC Global identification of O-GlcNAc transferase (OGT) interactors by a human proteome microarray and the construction of an OGT interactome. Proteomics 2014, 14, 1020-1030. [PubMed: 24536041]

23. Gao J, Yang Y, Qiu R, Zhang K, Teng X, Liu R \& Wang Y Proteomic analysis of the OGT interactome: novel links to epithelial-mesenchymal transition and metastasis of cervical cancer. Carcinogenesis 2018, 1-13. doi:10.1093/carcin/bgy097 [PubMed: 29342274]

24. Roux KJ, Kim DI, Raida M \& Burke B A promiscuous biotin ligase fusion protein identifies proximal and interacting proteins in mammalian cells. J. Cell Biol 2012, 196, 801-810. [PubMed: 22412018]

25. Samavarchi-Tehrani P, Samson R \& Gingras AC Proximity dependent biotinylation: Key enzymes and adaptation to proteomics approaches. Mol. Cell. Proteomics 2020, 19, 757-773. [PubMed: 32127388]

26. Elzi DJ, Song M, Hakala K, Weintraub ST \& Shiio Y Proteomic Analysis of the EWS-Fli - 1 Interactome Reveals the Role of the Lysosome in EWS-Fli - 1 Turnover. J. Proteome Res 2015,

27. Couzens A, Knight J, Kean M, Teo G, Weiss A, Dunham W, Lin Z, Bagshaw R, Sicheri F, Pawson T, Wrana J, Choi H \& Gingras A Protein Interaction Metwork of the Mammalian Hippo Pathway Reveals Mechanisms of Kinase-Phosphatase Interactions. Sci. Signal 2013, 6, 1-13.

28. Ramirez DH, Aonbangkhen C, Wu H-Y, Naftaly JA, Tang S, O'Meara TR \& Woo CM Engineering a proximity-directed O-GlcNAc transferase for selective protein O-GlcNAcylation in cells. ACS Chem. Biol 2020, 15, 1059-1066. [PubMed: 32119511]

29. Zhu Y \& Hart GW Targeting the O-GlcNAc Transferase to Specific Proteins Using RNA Aptamers. FASEB J. 2020, 31, 1-1.

30. Yu SH, Zhao P, Sun T, Gao Z, Moremen KW, Boons GJ, Wells L \& Steet R Selective exo-enzymatic labeling detects increased cell surface sialoglycoprotein expression upon megakaryocytic differentiation. J. Biol. Chem 2016, 291, 3982-3989. [PubMed: 26733198]

31. Weatherly DB, Atwood JA, Minning TA, Cavola C, Tarleton RL \& Orlando R A Heuristic Method for Assigning a False-discovery Rate for Protein Identifications from Mascot Database Search Results. Mol. Cell. Proteomics 2005, 4, 762-772. [PubMed: 15703444]

32. Zybailov B, Mosley AL, Sardiu ME, Coleman MK, Florens L \& Washburn MP Statistical analysis of membrane proteome expression changes in Saccharomyces cerevisiae. J. Proteome Res. 2006, 5, 2339-2347. [PubMed: 16944946]

33. UniProt Consortium T UniProt: A worldwide hub of protein knowledge. Nucleic Acids Res. 2019, 47, D506-D515. [PubMed: 30395287]

34. Schreiber E, Matthias P, Muller MM \& Schaffner W Rapid detection of octamer binding proteins with 'mini-extracts', prepared from a small number of cells. Nucleic Acids Res. 1989, 17, 6419. [PubMed: 2771659]

35. Gene Ontology Consortium T, Ashburner M, Ball CA, Blake JA, Botstein D, Butler H, Cherry JM, Davis AP, Dolinski K, Dwight SS, Eppig JT, Harris MA, Hill DP, Issel-Tarver L, Kasarskis A, Lewis S, Matese JC, Richardson JE, Ringwald M \& Rubin Gerald M. Sherlock G, The Gene Ontology Consortium, Ashburner1 Michael, Ball3 Catherine A., Blake4 Judith A., Botstein3 David, Butler1 Heather, Cherry3 J. Michael, Davis4 Allan P., Dolinski3 Kara, Dwight3 Selina S., Eppig4 Janan T., Harris3 Midori A., Hill4 David P., Laurie Is. Nat. Genet 2000, 25, 25-29. [PubMed: 10802651] 
36. Gene Ontology Consortium T The Gene Ontology Resource: 20 years and still GOing strong. Nucleic Acids Res. 2019, 47, D330-D338. [PubMed: 30395331]

37. Supek F, Bošnjak M, Škunca N \& Šmuc T Revigo summarizes and visualizes long lists of gene ontology terms. PLoS One 2011, 6,

38. Kuznetsova I, Lugmayr A, Siira SJ, Rackham O \& Filipovska A CirGO: An alternative circular way of visualising gene ontology terms. BMC Bioinformatics 2019, 20, 1-7. [PubMed: 30606105]

39. Online Mendelian In heritance in Man, OMIM. McKusick-Nathans Institute of Genetic Medicine, Johns Hopkins University (Baltimore, MD) https://omim.org/ (2020)

40. Alfaro JF, Gong C, Monroe ME, Aldrich JT, Clauss TRW, Purvine SO, Wang Z, Camp DG, Shabanowitz J, Stanley P, Hart GW, Hunt DF, Yang F \& Smith RD Tandem mass spectrometry identifies many mouse brain O -GlcNAcylated proteins including EGF domain-specific O GlcNAc transferase targets. Pnas 2012, 109, 7280-7285. [PubMed: 22517741]

41. Hornbeck PV, Zhang B, Murray B, Kornhauser JM, Latham V \& Skrzypek E PhosphoSitePlus, 2014: Mutations, PTMs and recalibrations. Nucleic Acids Res. 2015, 43, D512-D520. [PubMed: 25514926]

42. Lee JS \& Zhang Z O-linked N-acetylglucosamine transferase (OGT) interacts with the histone chaperone HIRA complex and regulates nucleosome assembly and cellular senescence. Proc. Natl. Acad. Sci. U. S. A 2016, 113, E3213-E3220. [PubMed: 27217568]

43. Slawson C, Lakshmanan T, Knapp S \& Hart GW A Mitotic GlcNAcylation/Phosphorylation Signaling Complex Alters the Posttranslational State of the Cytoskeletal Protein Vimentin. Mol. Biol. Cell 2008, 19, 4130-4140. [PubMed: 18653473]

44. Dey A, Seshasayee D, Noubade R, French DM, Chaurushiya MS, Kirkpatrick DS, Pham VC, Lill JR, Bakalarski E, Wu J, Phu L, Katavolos P, Saunders LM, Modrusan Z, Seshagiri S, Dong K, Lin Z, Martin F, Levine RL \& Dixit VM Loss of the tumor suppressor BAP1 causes myeloid transformation. Science (80-. ). 2012, 337, 1541-1546.

45. Kreppel LK \& Hart GW Regulation of a Cytosolic and Nuclear O-GlcNAc Transferase. J. Biol. Chem 1999, 274, 32015-32022. [PubMed: 10542233]

46. Groussaud D, Khair M, Tollenaere AI, Waast L, Kuo MS, Mangeney M, Martella C, Fardini Y, Coste S, Souidi M, Benit L, Pique C \& Issad T Hijacking of the O-GlcNAcZYME complex by the HTLV-1 Tax oncoprotein facilitates viral transcription. PLoS Pathog. 2017, 13, 1-23.

47. Gambetta MC, Oktaba K \& Muller J Essential Role of the Glycosyltransferase Sxc/Ogt in Polycomb Repression. Science (80-. ). 2009, 325, 93-96.

48. Sinclair DAR, Syrzycka M, Macauley MS, Rastgardani T, Komljenovic I, Vocadlo DJ, Brock HW \& Honda BM Drosophila O-GlcNAc transferase (OGT) is encoded by the Polycomb group (PcG) gene, super sex combs ( sxc). Proc. Natl. Acad. Sci. U. S. A 2009, 106, 13427-13432. [PubMed: 19666537]

49. Bullen JW, Balsbaugh JL, Chanda D, Shabanowitz J, Hunt DF, Neumann D \& Hart GW Crosstalk between two essential nutrient-sensitive enzymes O-GlcNAc Transferase (OGT) and AmpActivated Protein Kinase (AMPK). J. Biol. Chem 2014, 289, 10592-10606. [PubMed: 24563466]

50. Wu D, Zhao L, Feng Z, Yu C, Ding J, Wang L, Wang F, Liu D, Zhu H, Xing F, Conaway JW, Conaway RC, Cai Y \& Jin J O-Linked N-acetylglucosamine transferase 1 regulates global histone H4 acetylation via stabilization of the nonspecific lethal protein NSL3. J. Biol. Chem 2017, 292, 10014-10025. [PubMed: 28450392]

51. Hrit J, Goodrich L, Li C, Wang B-A, Nie J, Cui X, Martin EA, Simental E, Fernandez J, Liu MY, Nery JR, Castanon R, Kohli RM, Tretyakova N, He C, Ecker JR, Goll M \& Panning B OGT binds a conserved C-terminal domain of TET1 to regulate TET1 activity and function in development. Elife 2018, 7, 1-23.

52. Sakabe K, Wang Z \& Hart GW $\beta$-N-acetylglucosamine (O-GlcNAc) is part of the histone code. Proc. Natl. Acad. Sci. U. S. A 2010, 107, 19915-19920. [PubMed: 21045127]

53. Brownlee PM, Meisenberg C \& Downs JA The SWI/SNF chromatin remodelling complex: Its role in maintaining genome stability and preventing tumourigenesis. DNA Repair (Amst). 2015, 32, 127-133. [PubMed: 25981841]

54. Huang L, Jolly LA, Willis-Owen S, Gardner A, Kumar R, Douglas E, Shoubridge C, Wieczorek D, Tzschach A, Cohen M, Hackett A, Field M, Froyen G, Hu H, Haas SA, Ropers HH, Kalscheuer 
VM, Corbett MA \& Gecz J A noncoding, regulatory mutation implicates HCFC1 in nonsyndromic intellectual disability. Am. J. Hum. Genet 2012, 91, 694-702. [PubMed: 23000143]

55. Kreppel LK, Blomberg M. a \& Hart GW Dynamic Glycosylation of Nuclear and Cytosolic Proteins. J. Biol. Chem 1997, 272, 9308-9315. [PubMed: 9083067]

56. Martinez MR, Dias TB, Natov PS \& Zachara NE Stress-Induced O-GlcNAcylation, an Adaptive Process of Injured Cells. Biochem. Soc. Trans 2017, 45, 237-249. [PubMed: 28202678]

57. Kaasik K, Kivimae S, Allen JJ, Chalkley RJ, Huang Y, Baer K, Kissel H, Burlingame AL, Shokat KM, Ptacek LJ \& Fu Y-H Glucose Sensor O-GlcNAcylation Coordinates with Phosphorylation to Regulate Circadian Clock. Cell Metab. 2013, 17, 291-302. [PubMed: 23395175]

58. Kelly WG, Dahmus ME \& Hart GW RNA polymerase II is a glycoprotein. J. Biol. Chem 1993, 268, 10416-10424. [PubMed: 8486697]

59. Ranuncolo SM, Ghosh S, Hanover JA, Hart GW \& Lewis BA Evidence of the involvement of O-GlcNAc-modified human RNA polymerase II CTD in transcription in vitro and in vivo. J. Biol. Chem 2012, 287, 23549-23561. [PubMed: 22605332]

60. Eustice M, Bond MR \& Hanover JA O-GlcNAc cycling and the regulation of nucleocytoplasmic dynamics. Biochem. Soc. Trans 2017, 45, 427-436. [PubMed: 28408483]

61. Wang Q, Fang P, He R, Li M, Yu H, Zhou L, Yi Y, Wang F, Rong Y, Zhang Y, Chen A, Peng N, Lin Y, Lu M, Zhu Y, Peng G, Rao L \& Liu S O-GlcNAc transferase promotes influenza A virus-induced cytokine storm by targeting interferon regulatory factor-5. Sci. Adv 2020, 6, 1-13.

62. Baldini SF, Steenackers A, Olivier-Van Stichelen S, Mir AM, Mortuaire M, Lefebvre T \& Guinez C Glucokinase expression is regulated by glucose through O-GlcNAc glycosylation. Biochem. Biophys. Res. Commun 2016, 478, 942-948. [PubMed: 27520373]

63. Yi W, Clark PM, Mason DE, Keenan MC, Hill C, William A., Iii, G., Peters EC, Driggers EM \& Hsieh-wilson LC PFK1 Glycosylation Is a Key Regulator of Cancer Cell Growth and Central Metabolic Pathways Wen. Science (80-. ). 2013, 337, 975-980.

64. Olivier-Van Stichelen S, Dehennaut V, Buzy A, Zachayus JL, Guinez C, Mir AM, El YazidiBelkoura I, Copin MC, Boureme D, Loyaux D, Ferrara P \& Lefebvre T O-GlcNAcylation stabilizes $\beta$-catenin through direct competition with phosphorylation at threonine 41 . FASEB J. 2014, 28, 3325-3328. [PubMed: 24744147]

65. Hanover JA, Chen W \& Bond MR O- GlcNAc in cancer : An Oncometabolism-fueled vicious cycle. J. Bioenerg. Biomembr 2018, 50, 155-173. [PubMed: 29594839]

66. Akan I, Stichelen SO-V, Bond MR \& Hanover JA Nutrient-driven O-GlcNAc in proteostasis and neurodegeneration. J. Neurochem 2017, 12, 3218-3221.

67. Pravata VM, Omelková M, Stavridis MP, Desbiens CM, Stephen HM, Lefeber DJ, Gecz J, Gundogdu M, Õnap K, Joss S, Schwartz CE, Wells L \& van Aalten DMF An intellectual disability syndrome with single-nucleotide variants in O-GlcNAc transferase. Eur. J. Hum. Genet 2020, 28, 706-714. [PubMed: 32080367]

68. Danino YM, Even D, Ideses D \& Juven-Gershon T The core promoter: At the heart of gene expression. Biochim. Biophys. Acta - Gene Regul. Mech 2015, 1849, 1116-1131.

69. Joiner CM, Levine ZG, Aonbangkhen C, Woo CM \& Walker S Aspartate residues far from the active site drive O-GlcNAc transferase substrate selection. J. Am. Chem. Soc 2019, jacs.9b06061. doi:10.1021/jacs.9b06061

70. Lazarus MB, Nam Y, Jiang J, Sliz P \& Walker S Structure of human O-GlcNAc transferase and its complex with a peptide substrate. Nature2 2011, 469, 564-567.

71. Zeidan Q, Wang Z, Maio A. De\& Hart GW O-GlcNAc Cycling Enzymes Associate with the Translational Machinery and Modify Core Ribosomal Proteins. Mol. Biol. Cell 2010, 21, 19221936. [PubMed: 20410138]

72. Selvan N, George S, Serajee FJ, Shaw M, Hobson L, Kalscheuer V, Prasad N, Levy SE, Taylor J, Aftimos S, Schwartz CE, Huq AHMM, Gecz J \& Wells L Characterization of missense mutations in the tetratricopeptide region of O-GlcNAc transferase causal for X-linked intellectual disability. J. Biol. Chem 2018, 
A

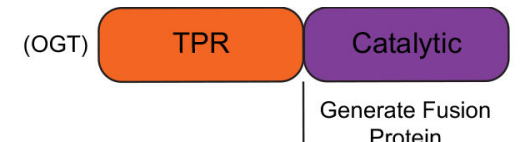

Protein

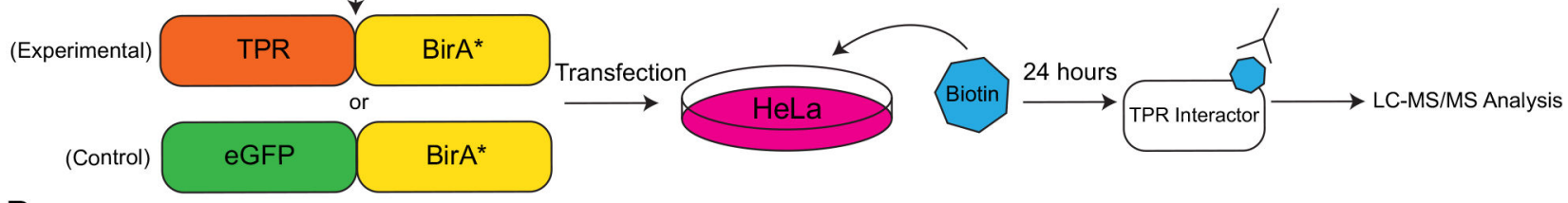

B

\section{1: Peptide Selection:}

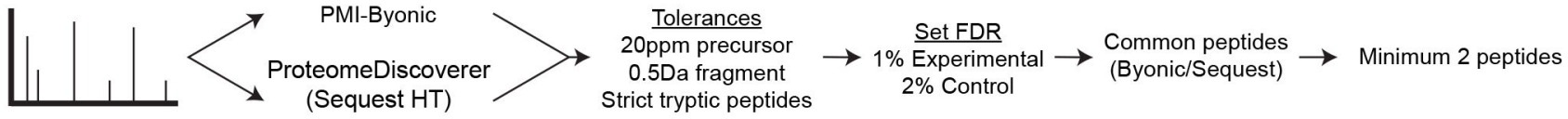

\section{2: Statistical Analysis $(n=3)$ :}

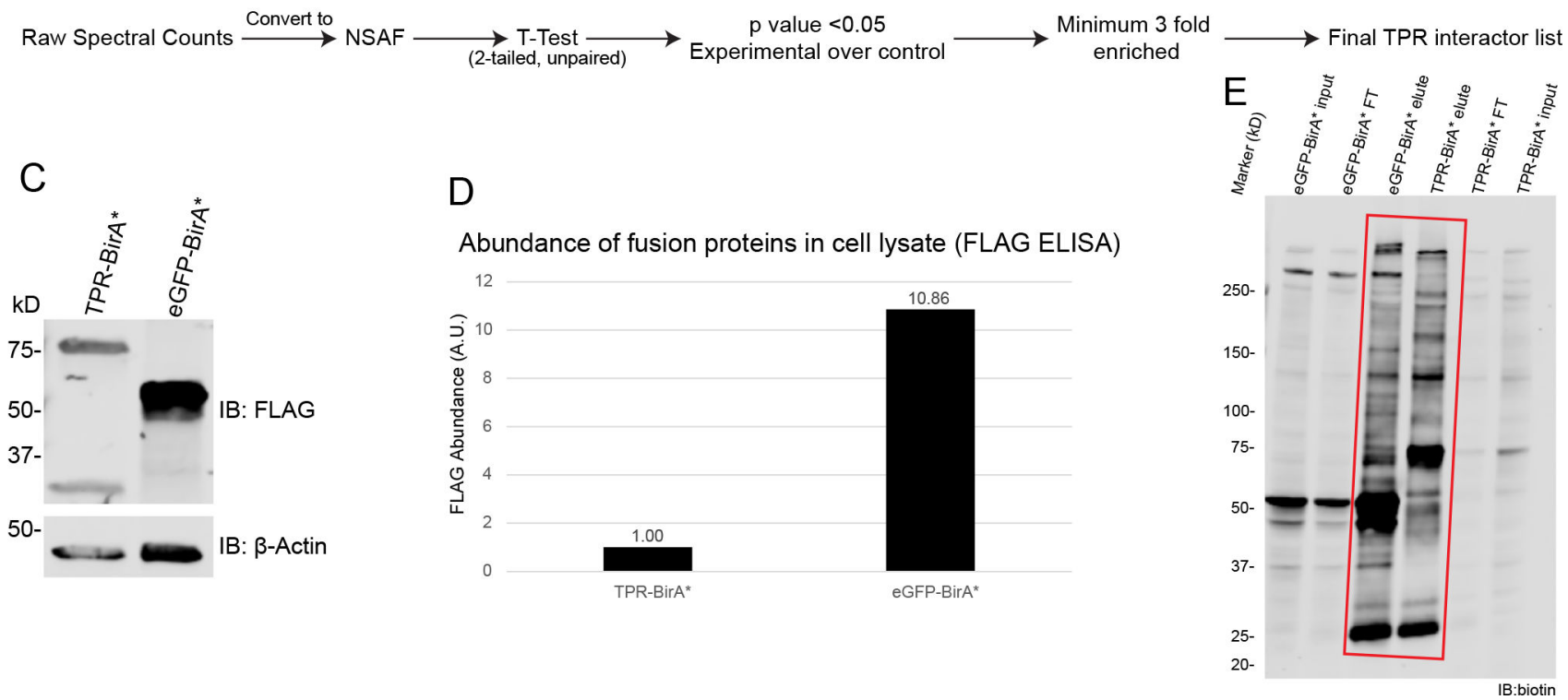

Figure 1 : BioID approach to define OGT TPR interactors in HeLa cells.

$\boldsymbol{A}$, Workflow of BioID process for identifying TPR interactors. $\boldsymbol{B}$, Workflow of MS analysis and protein validation. Samples were prepped in biological triplicate. $\boldsymbol{C}$, Western blot showing expression of TPR-BirA* and eGFP-BirA* fusion proteins (anti-FLAG tag), $\beta$-actin as loading control. 20ug/lane. D, ELISA quantification of FLAG-tagged fusion proteins (one representative replicate, A.U.=Arbitrary units) $\boldsymbol{E}$, Representative western blot with anti-biotin antibody demonstrating enrichment of biotinylated proteins following biotin immunoprecipitation (representative blot from three replicates). Red box indicates lanes

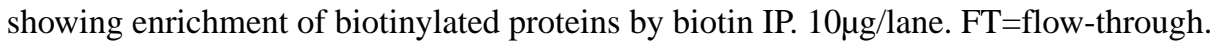
Forelute and FT, 10ug/lane. Forelute, 25\% of total eluate loaded. 


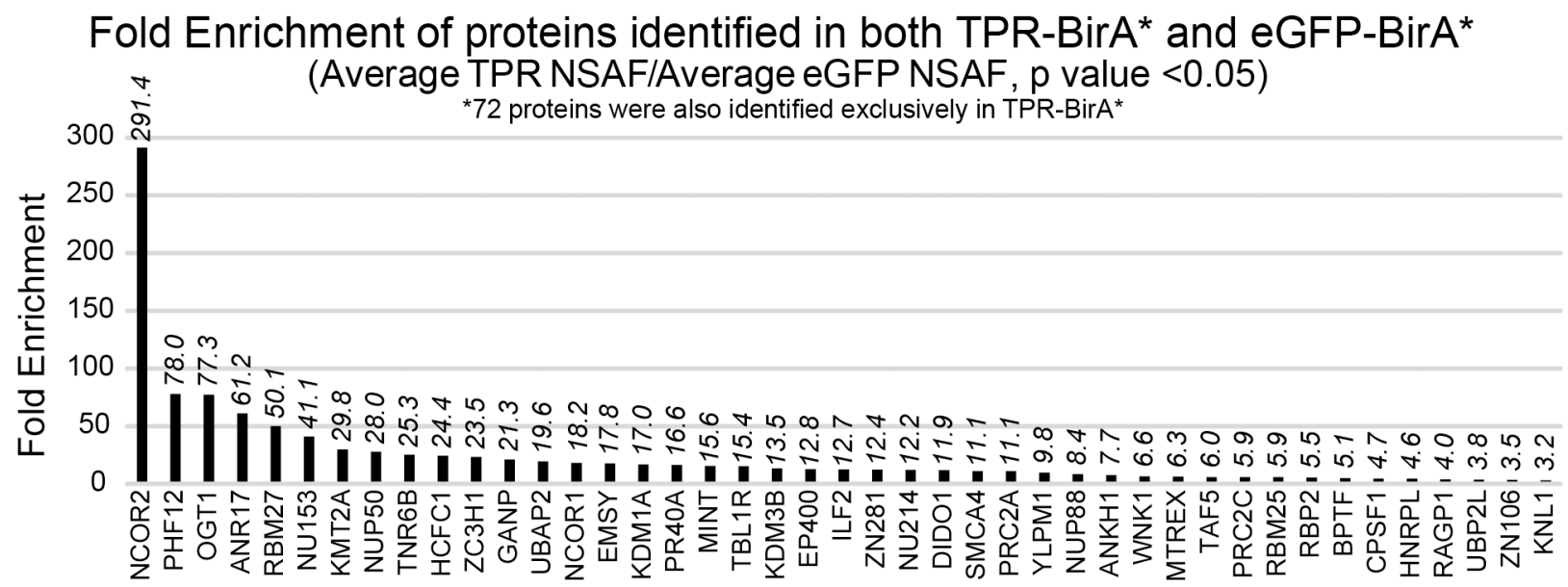

Figure 2: Proteins identified in both TPR-BirA* and eGFP-BirA* are enriched in TPR-BirA*. Fold enrichment values for all proteins identified in both TPR-BirA* and eGFP-BirA*. Fold enrichment values are average NSAF of TPR-BirA* over average NSAF of eGFP-BirA*. Note that 72 proteins were only observed in TPR-BirA*. 
A OGT catalytic domain peptide: LVSIVADQLEK

Theoretical mass: $1214.6991 \mathrm{Da}$

Average Mass Error: $\Delta \mathbf{2 . 1 7 p p m}$

Fold increase: 11.7 (Average $9.1+/-2.3$ )

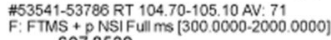

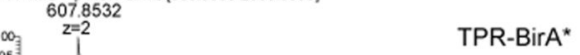

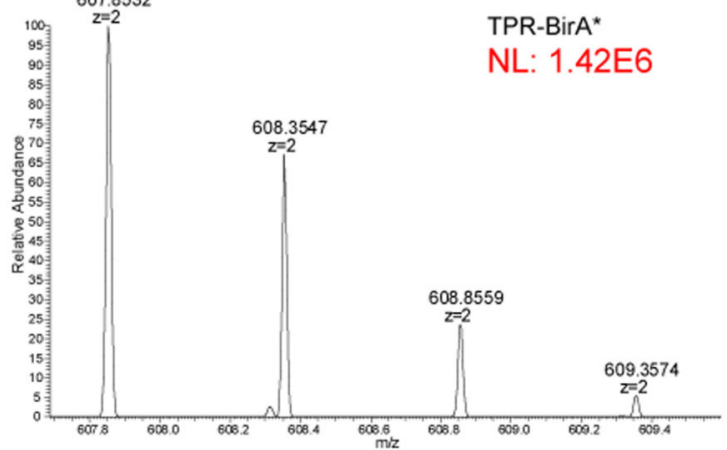

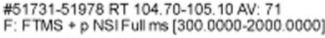

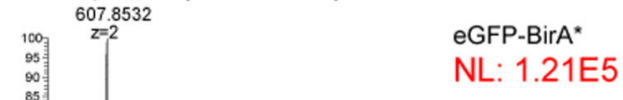

$\mathrm{NL}: 1.21 \mathrm{E} 5$

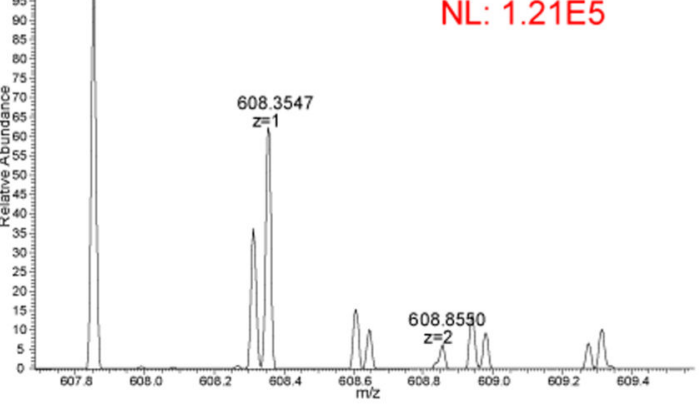

B HCF-1 peptide: IATGHGQQGVTQVVLK

Theoretical mass: $1635.9177 \mathrm{Da}$ Average Mass Error: $\Delta 0.749 \mathrm{ppm}$

Fold increase: 26.3 (Average $36.4+/-24.4$ )

\#27673-27973 RT 59.00-59.80 AV: 173
F: FTMS p N NIF Uul ms [300.0000-2000.0000]

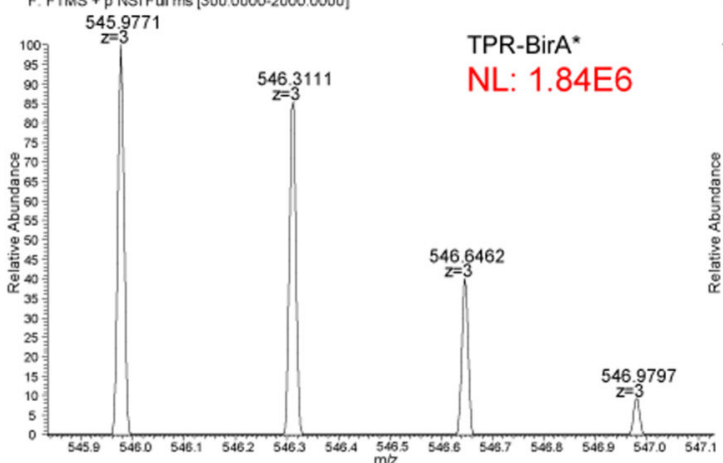

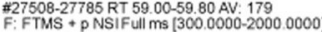

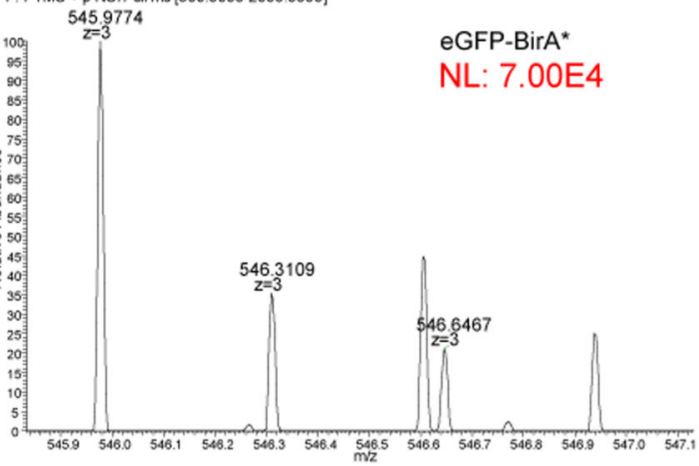

C KNL1 peptide: AQVESCQLNNR (57.021 (C6))

Theoretical mass: $1318.6168 \mathrm{Da}$

Average Mass Error: $\Delta \mathbf{2 . 1 2 \mathrm { ppm }}$

Fold increase: 3.08 (Average 3.93 +/- 1.93)

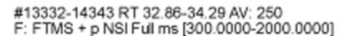

659.8123

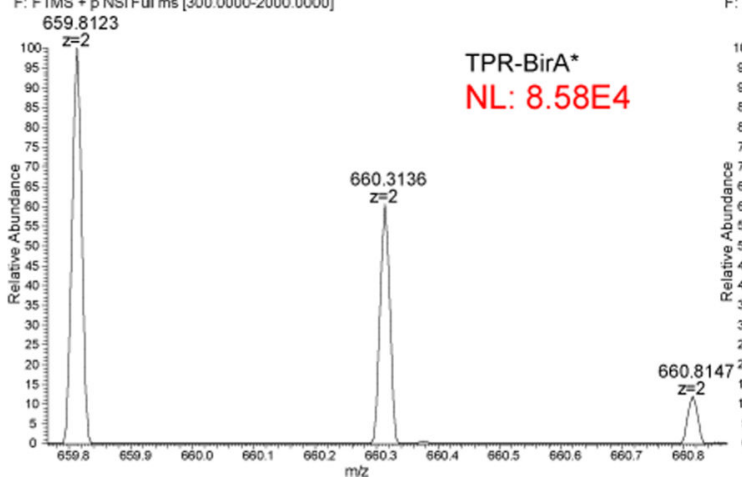

M1 13951-15007 RT $32.86 .34 .29 \mathrm{AV}: 236$
F: FTMS + p NSIFul ms [300.0000-2000.0000]

$$
\begin{gathered}
659.8126 \\
Z=2
\end{gathered}
$$

${ }_{100}^{659.8} \mathrm{z}=2$

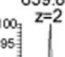

GFP-BirA*

$\mathrm{NL}: 2.81 \mathrm{E} 4$

Figure 3: Reconstructed ion chromatograms confirm peptide-level enrichment of TPR-BirA* interactors.

Fold increase is for the replicate shown. The average fold intensity for that peptide (averaged across all replicates and fractions in which that peptide appeared) is shown with the standard deviation. Average mass error is the absolute value of the mean across 3 replicates of both TPR-BirA* and eGFP-BirA*. NL=Normalization Level (Base Peak Intensity) $\boldsymbol{A}$, Rectonstructed ion chromatograms demonstrating greater enrichment of a catalytic OGT peptide in TPR-BirA* compared to eGFP-BirA*. B, Reconstructed ion chromatograms 
demonstrating greater enrichment of an FICF-1 peptide in TPR-BirA* compared to eGFPBirA*. $\boldsymbol{C}$, Reconstructed ion chromatograms demonstrating greater enrichment of a KNL1 peptide (novel interactor closest to cut-off for assignment) in TPR-BirA* compared to eGFP-BirA*. 
A

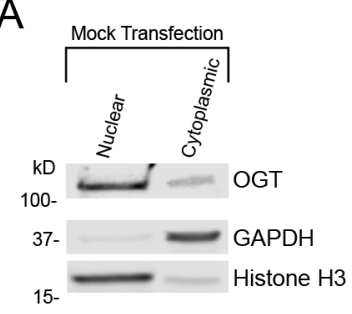

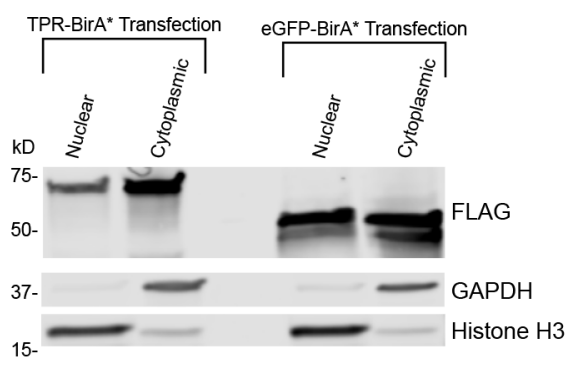

B

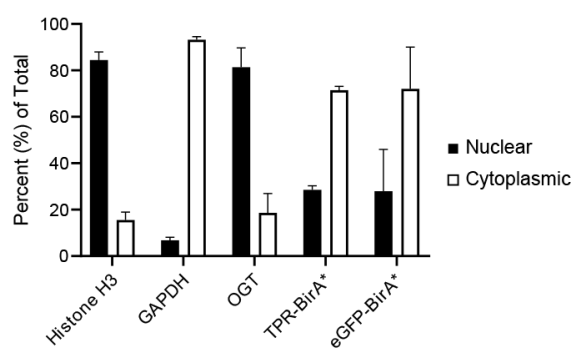

C
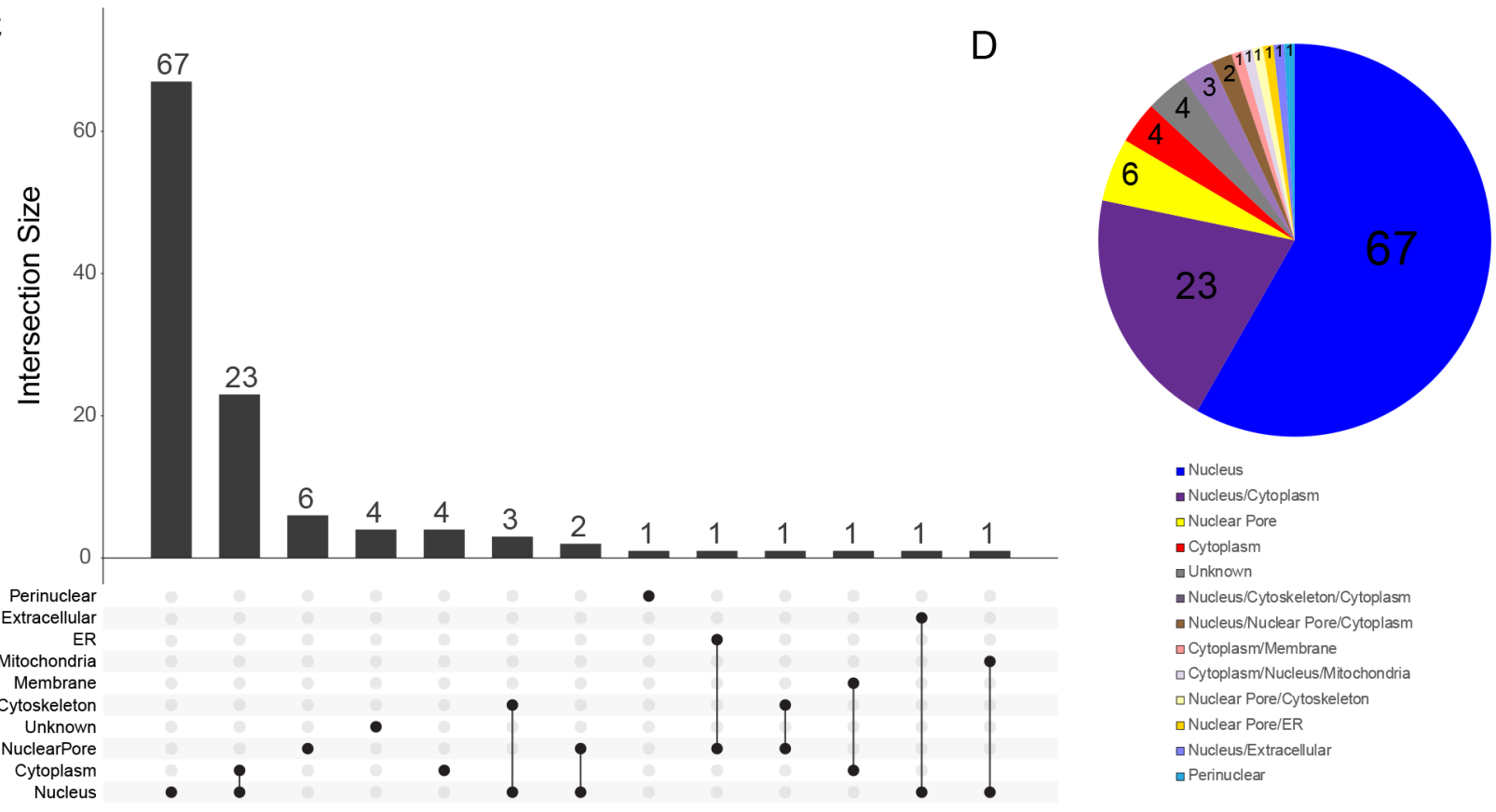

- Nucleus

- Nucleus/Cytoplasm

a Nuclear Pore

- Cytoplasm

๑nknown

- Nucleus/Cytoskeleton/Cytoplasm

- Nucleus/Nuclear Pore/Cytoplasm

$\square$ Cytoplasm/Membrane

$\square$ Cytoplasm/Nucleus/Mitochondria

$\square$ Nuclear Pore/Cytoskeleton

$\square$ Nuclear Pore/ER

- Nucleus/Extracellular

$\square$ Perinuclear

Figure 4: TPR interactors are primarily nuclear localized.

$A$, Subcellular fractionation of HeLa cells demonstrating localization of OGT (anti-OGT F12) and BirA* fusion proteins (anti-FLAG tag). Cytoplasmic marker is GAPDH, nuclear marker is Histone H3. 10ug/lane, representative western blot of three biological replicates $\boldsymbol{B}$, Ratios of nuclear to cytoplasmic expression of marker proteins (Nuclear: Histone H3, Cytoplasmic: GAPDH) and fusion proteins. Averaged across three biological replicates. $\boldsymbol{C}$, UpsetR plot showing the subcellular localization of TPR interactors. $\boldsymbol{D}$, Venn diagram showing the subcellular localization of TPR interactors. Numbers represent the total number of TPR interactors in that category. Localization determined using UniProt. 


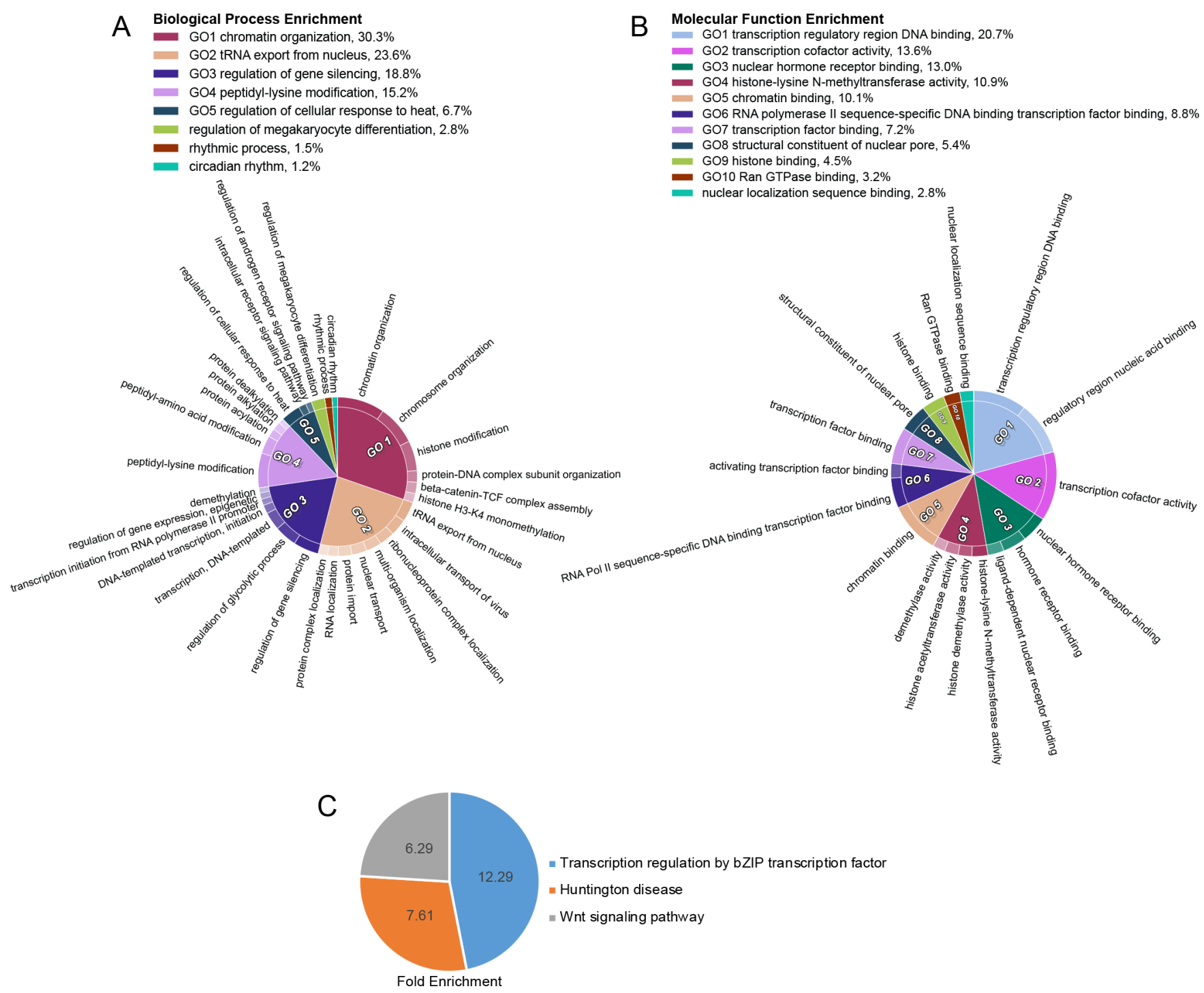

Figure 5: TPR interactors demonstrate enrichment in biological processes and disease states. $\boldsymbol{A}$, CirGo plot showing enriched biological processes in the TPR protein interactor list $\boldsymbol{B}$, CirGo plot showing enriched molecular functions in the TPR protein interactor list $\boldsymbol{C}$, Venn diagram of PANTHER Pathway enrichment of TPR interactors. Numbers are the fold enrichment of the pathway process over expected enrichment. 
A

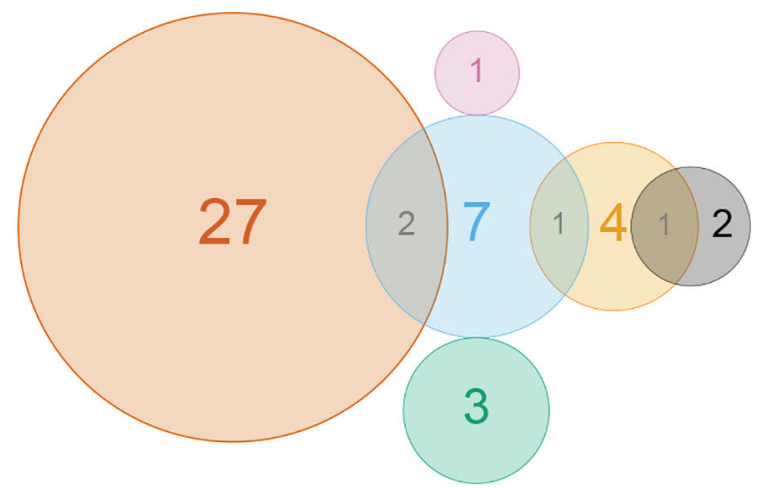

Intellectual Disability Immunodeficiency Malignancy

Congenital, other Neurological, other Hormone

B

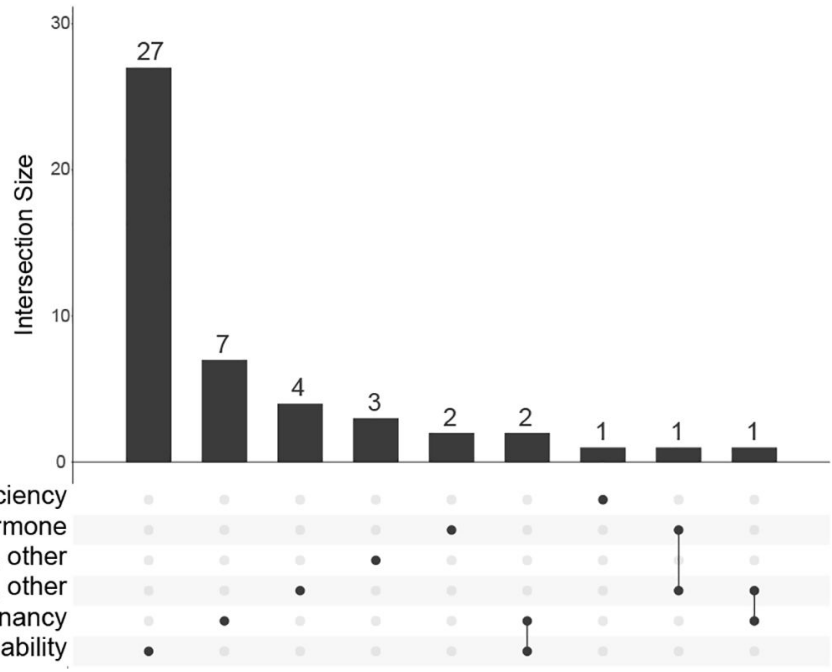

Figure 6: OGT interacts with proteins involved in intellectual disability in HeLa cells.

$\boldsymbol{A}$, Venn diagram showing disease states enriched in the TPR interactors. $\boldsymbol{B}$, UpsetR plot showing disease states enriched in the TPR interactors. Disease associations determined using the OMIM resource. 
Table 1:

Interactors identified as TPR-BirA* interactors in HeLa cells

\begin{tabular}{|c|c|}
\hline Uniprot Accession & Sequence Name \\
\hline ZHX3_HUMAN & Zinc fingers and homeoboxes protein 3 \\
\hline CCAR1_HUMAN & Cell division cycle and apoptosis regulator protein 1 \\
\hline JHD2C_HUMAN & Probable JmjC domain-containing histone demethylation protein $2 C$ \\
\hline ZN609_HUMAN & Zinc finger protein 609 \\
\hline QSER1_HUMAN & Glutamine and serine-rich protein 1 \\
\hline RBM26_HUMAN & RNA-binding protein 26 \\
\hline ZHX2_HUMAN & Zinc fingers and homeoboxes protein 2 \\
\hline PRR12_HUMAN & Proline-rich protein 12 \\
\hline TRPS1_HUMAN & Zinc finger transcription factor Trps 1 \\
\hline BCOR_HUMAN & $B C L-6$ corepressor \\
\hline OGA_HUMAN & Protein O-GlcNAcase ${ }^{43}$ \\
\hline CIC_HUMAN & Protein capicua homolog \\
\hline KMT2C_HUMAN & Histone-lysine $\mathrm{N}$-methyltransferase $2 \mathrm{C}$ \\
\hline CEP85_HUMAN & Centrosomal protein of $85 \mathrm{kDa}$ \\
\hline NCOA6_HUMAN & Nuclear receptor coactivator 6 \\
\hline HIRA_HUMAN & Protein HIRA ${ }^{42}$ \\
\hline ARI5B_HUMAN & AT-rich interactive domain-containing protein $5 B$ \\
\hline ZN318_HUMAN & Zinc finger protein 318 \\
\hline RGPD8_HUMAN & RANBP2-like and GRIP domain-containing protein 8 \\
\hline RGPD5_HUMAN & RANBP2-like and GRIP domain-containing protein 5/6 \\
\hline ZFR_HUMAN & Zinc finger RNA-binding protein \\
\hline RGPD3_HUMAN & RanBP2-like and GRIP domain-containing protein 3 \\
\hline ZC3HE_HUMAN & Zinc finger $\mathrm{CCCH}$ domain-containing protein 14 \\
\hline BAP1_HUMAN & Ubiquitin carboxyl-terminal hydrolase BAP1 44 \\
\hline TET2_HUMAN & Methylcytosine dioxygenase TET2 ${ }^{20}$ \\
\hline ZEP1_HUMAN & Zinc finger protein 40 \\
\hline RPRD2_HUMAN & Regulation of nuclear pre-mRNA domain-containing protein 2 \\
\hline PHC3_HUMAN & Polyhomeotic-like protein 3 \\
\hline CABIN_HUMAN & Calcineurin-binding protein cabin-1 \\
\hline SET1A_HUMAN & Histone-lysine N-methyltransferase SETD1A \\
\hline TBL1X_HUMAN & F-box-like/WD repeat-containing protein TBL1X \\
\hline MEF2D_HUMAN & Myocyte-specific enhancer factor $2 D$ \\
\hline NUP62_HUMAN & Nuclear pore glycoprotein p62 \\
\hline PCF11_HUMAN & Pre-mRNA cleavage complex 2 protein Pcf11 \\
\hline TAF4_HUMAN & Transcription initiation factor TFIID subunit 4 \\
\hline LIN54_HUMAN & Protein lin-54 homolog \\
\hline
\end{tabular}




\begin{tabular}{|c|c|}
\hline Uniprot Accession & Sequence Name \\
\hline UBN2_HUMAN & Ubinuclein-2 \\
\hline P121C_HUMAN & Nuclear envelope pore membrane protein POM 121C \\
\hline RESF1_HUMAN & Retroelement silencing factor 1 \\
\hline TASO2_HUMAN & Protein TASOR 2 \\
\hline DOT1L_HUMAN & Histone-lysine N-methyltransferase, H3 lysine-79 specific \\
\hline NUP58_HUMAN & Nucleoporin p58/p45 \\
\hline ASXL2_HUMAN & Putative Polycomb group protein ASXL2 \\
\hline RBM33_HUMAN & RNA-binding protein 33 \\
\hline SMRC1_HUMAN & SWI/SNF complex subunit SMARCC1 \\
\hline ICE1_HUMAN & Little elongation complex subunit 1 \\
\hline PRC2B_HUMAN & Protein PRRC2B \\
\hline NUP54_HUMAN & Nucleoporin p54 \\
\hline SIN3A_HUMAN & Paired amphipathic helix protein Sin $3 a^{19}$ \\
\hline ARI1B_HUMAN & AT-rich interactive domain-containing protein 1B \\
\hline CBP_HUMAN & $C R E B$-binding protein \\
\hline SMRC2_HUMAN & SWI/SNF complex subunit SMARCC2 \\
\hline LRIF1_HUMAN & Ligand-dependent nuclear receptor-interacting factor 1 \\
\hline KDM2B_HUMAN & Lysine-specific demethylase $2 \mathrm{~B}$ \\
\hline NCOA2_HUMAN & Nuclear receptor coactivator 2 \\
\hline UBN1_HUMAN & Ubinuclein-1 $^{42}$ \\
\hline KMT2D_HUMAN & Histone-lysine $\mathrm{N}$-methyltransferase $2 \mathrm{D}$ \\
\hline ZHX1_HUMAN & Zinc fingers and homeoboxes protein 1 \\
\hline PF21A_HUMAN & PHD finger protein 21A \\
\hline ARI1A_HUMAN & AT-rich interactive domain-containing protein $1 \mathrm{~A}$ \\
\hline ZFHX3_HUMAN & Zinc finger homeobox protein 3 \\
\hline EP300_HUMAN & Histone acetyltransferase $\mathrm{p} 300$ \\
\hline SF3A1_HUMAN & Splicing factor $3 \mathrm{~A}$ subunit 1 \\
\hline BCL9_HUMAN & B-cell CLL/lymphoma 9 protein \\
\hline TRRAP_HUMAN & Transformation/transcription domain-associated protein \\
\hline ACL6A_HUMAN & Actin-like protein $6 \mathrm{~A}$ \\
\hline TAF6_HUMAN & Transcription initiation factor TFIID subunit 6 \\
\hline TNR6A_HUMAN & Trinucleotide repeat-containing gene $6 A$ protein \\
\hline POGZ_HUMAN & Pogo transposable element with ZNF domain \\
\hline AHDC1_HUMAN & AT-hook DNA-binding motif-containing protein 1 \\
\hline I2BP2_HUMAN & Interferon regulatory factor 2-binding protein 2 \\
\hline RREB1_HUMAN & Ras-responsive element-binding protein 1 \\
\hline NCOR2_HUMAN & Nuclear receptor corepressor 2 \\
\hline PHF12_HUMAN & PHD finger protein 12 \\
\hline OGT1_HUMAN & UDP-N-acetylglucosamine--peptide $N$-acetylglucosaminyltransferase 110 kDa subunit ${ }^{45}$ \\
\hline
\end{tabular}




\begin{tabular}{|c|c|}
\hline Uniprot Accession & Sequence Name \\
\hline ANR17_HUMAN & Ankyrin repeat domain-containing protein 17 \\
\hline RBM27_HUMAN & $R N A$-binding protein 27 \\
\hline NU153_HUMAN & Nuclear pore complex protein Nup153 \\
\hline KMT2A_HUMAN & Histone-lysine N-methyltransferase $2 \mathrm{~A}$ \\
\hline NUP50_HUMAN & Nuclear pore complex protein Nup50 \\
\hline TNR6B_HUMAN & Trinucleotide repeat-containing gene $6 \mathrm{~B}$ protein \\
\hline HCFC1_HUMAN & Host cell factor $1^{21}$ \\
\hline ZC3H1_HUMAN & Zinc finger $\mathrm{C} 3 \mathrm{H} 1$ domain-containing protein \\
\hline GANP_HUMAN & Germinal-center associated nuclear protein \\
\hline UBAP2_HUMAN & Ubiquitin-associated protein 2 \\
\hline NCOR1_HUMAN & Nuclear receptor corepressor 1 \\
\hline EMSY_HUMAN & BRCA2-interacting transcriptional repressor EMSY \\
\hline KDM1A_HUMAN & Lysine-specific histone demethylase $1 \mathrm{~A}$ \\
\hline PR40A_HUMAN & Pre-mRNA-processing factor 40 homolog A \\
\hline MINT_HUMAN & Msx2-interacting protein \\
\hline TBL1R_HUMAN & F-box-like/WD repeat-containing protein TBL1XR1 \\
\hline KDM3B_HUMAN & Lysine-specific demethylase 3B \\
\hline EP400_HUMAN & E1A-binding protein $p 400$ \\
\hline ILF2_HUMAN & Interleukin enhancer-binding factor 2 \\
\hline ZN281_HUMAN & Zinc finger protein 281 \\
\hline NU214_HUMAN & Nuclear pore complex protein Nup214 \\
\hline DIDO1_HUMAN & Death-inducer obliterator 1 \\
\hline SMCA4_HUMAN & Transcription activator BRG1 \\
\hline PRC2A_HUMAN & Protein PRRC2A \\
\hline YLPM1_HUMAN & YLP motif-containing protein 1 \\
\hline NUP88_HUMAN & Nuclear pore complex protein Nup 88 \\
\hline ANKH1_HUMAN & Ankyrin repeat and $\mathrm{KH}$ domain-containing protein 1 \\
\hline WNK1_HUMAN & Serine/threonine-protein kinase $W N K 1$ \\
\hline TAF5_HUMAN & Transcription initiation factor TFIID subunit 5 \\
\hline PRC2C_HUMAN & Protein PRRC2C \\
\hline RBM25_HUMAN & RNA-binding protein 25 \\
\hline RBP2_HUMAN & E3 SUMO-protein ligase RanBP2 \\
\hline BPTF_HUMAN & Nucleosome-remodeling factor subunit BPTF \\
\hline CPSF1_HUMAN & Cleavage and polyadenylation specificity factor subunit 1 \\
\hline HNRPL_HUMAN & Heterogeneous nuclear ribonucleoprotein $\mathrm{L}$ \\
\hline RAGP1_HUMAN & Ran GTPase-activating protein 1 \\
\hline UBP2L_HUMAN & Ubiquitin-associated protein 2-like \\
\hline ZN106_HUMAN & Zinc finger protein 106 \\
\hline KNL1_HUMAN & Kinetochore scaffold 1 \\
\hline
\end{tabular}


Legend:

Black Accession: ID'd only in TPR

Bold Accession: ID'd in both TPR and eGFP, p value $<0.05$

Italicized Protein Name: Known O-GlcNAc modified

Bold and Italicized Protein Name: Known OGT interactors (with citations) 
Table 2:

Reactome pathways enriched in TPR interactors list (FDR <5E-9)

\begin{tabular}{|c|c|c|c|c|c|}
\hline Reactome Pathway & Identifier & $\begin{array}{c}\text { Protein } \\
\text { Number }\end{array}$ & Fold enrichment & $P$ value & FDR \\
\hline Chromatin organization & R-HSA-4839726 & 27 & 20.4 & $9.70 \mathrm{E}-27$ & $1.11 \mathrm{E}-23$ \\
\hline Chromatin modifying enzymes & R-HSA-3247509 & 27 & 20.4 & $9.70 \mathrm{E}-27$ & $2.22 \mathrm{E}-23$ \\
\hline SUMO E3 ligases SUMOylate target proteins & R-HSA-3108232 & 15 & 17.32 & $3.26 \mathrm{E}-14$ & $1.86 \mathrm{E}-11$ \\
\hline SUMOylation & R-HSA-2990846 & 15 & 16.69 & $5.42 \mathrm{E}-14$ & $2.47 \mathrm{E}-11$ \\
\hline Transcriptional regulation by RUNX1 & R-HSA-8878171 & 16 & 14.22 & $7.48 \mathrm{E}-14$ & $2.85 \mathrm{E}-11$ \\
\hline Rev-mediated nuclear export of HIV RNA & R-HSA-165054 & 9 & 50.99 & $9.89 \mathrm{E}-13$ & $3.23 \mathrm{E}-10$ \\
\hline HCMV Early Events & R-HSA-9609690 & 12 & 21.76 & $1.15 \mathrm{E}-12$ & $3.29 \mathrm{E}-10$ \\
\hline Interactions of Rev with host cellular proteins & R-HSA-177243 & 9 & 47.99 & $1.58 \mathrm{E}-12$ & $4.00 \mathrm{E}-10$ \\
\hline $\begin{array}{l}\text { Transport of Mature mRNA Derived from an Intronless } \\
\text { Transcript }\end{array}$ & R-HSA-159231 & 9 & 41.84 & $4.58 \mathrm{E}-12$ & $1.05 \mathrm{E}-09$ \\
\hline $\begin{array}{l}\text { Transport of Mature mRNAs Derived from Intronless } \\
\text { Transcripts }\end{array}$ & R-HSA-159234 & 9 & 40.8 & $5.59 \mathrm{E}-12$ & $1.16 \mathrm{E}-09$ \\
\hline SUMOylation of DNA replication proteins & R-HSA-4615885 & 9 & 37.95 & $9.87 \mathrm{E}-12$ & $1.61 \mathrm{E}-09$ \\
\hline Processing of Capped Intron-Containing Pre-mRNA & R-HSA-72203 & 15 & 11.43 & $9.06 \mathrm{E}-12$ & $1.72 \mathrm{E}-09$ \\
\hline SUMOylation of RNA binding proteins & R-HSA-4570464 & 9 & 37.95 & $9.87 \mathrm{E}-12$ & $1.73 \mathrm{E}-09$ \\
\hline HCMV Infection & R-HSA-9609646 & 12 & 17.55 & $1.18 \mathrm{E}-11$ & $1.80 \mathrm{E}-09$ \\
\hline Transport of Ribonucleoproteins into the Host Nucleus & R-HSA-168271 & 8 & 50.02 & $2.16 \mathrm{E}-11$ & $2.60 \mathrm{E}-09$ \\
\hline NEP/NS2 Interacts with the Cellular Export Machinery & R-HSA-168333 & 8 & 50.02 & $2.16 \mathrm{E}-11$ & $2.75 \mathrm{E}-09$ \\
\hline $\begin{array}{l}\text { Defective TPR may confer susceptibility towards } \\
\text { thyroid papillary carcinoma (TPC) }\end{array}$ & R-HSA-5619107 & 8 & 50.02 & $2.16 \mathrm{E}-11$ & 2.91E-09 \\
\hline $\begin{array}{l}\text { Regulation of Glucokinase by Glucokinase Regulatory } \\
\text { Protein }\end{array}$ & R-HSA-170822 & 8 & 50.02 & $2.16 \mathrm{E}-11$ & 3.09E-09 \\
\hline Export of Viral Ribonucleoproteins from Nucleus & R-HSA-168274 & 8 & 48.35 & $2.73 \mathrm{E}-11$ & 3.12E-09 \\
\hline Vpr-mediated nuclear import of PICs & R-HSA-180910 & 8 & 46.79 & $3.42 \mathrm{E}-11$ & 3.39E-09 \\
\hline Late Phase of HIV Life Cycle & R-HSA-162599 & 12 & 16 & $3.23 \mathrm{E}-11$ & $3.51 \mathrm{E}-09$ \\
\hline Nuclear import of Rev protein & R-HSA-180746 & 8 & 46.79 & $3.42 \mathrm{E}-11$ & $3.55 \mathrm{E}-09$ \\
\hline SUMOylation of SUMOylation proteins & R-HSA-4085377 & 8 & 45.33 & $4.25 \mathrm{E}-11$ & $3.89 \mathrm{E}-09$ \\
\hline Transport of the SLBP independent Mature mRNA & R-HSA-159227 & 8 & 45.33 & $4.25 \mathrm{E}-11$ & 4.05E-09 \\
\hline Nuclear Pore Complex (NPC) Disassembly & R-HSA-3301854 & 8 & 43.95 & $5.26 \mathrm{E}-11$ & $4.29 \mathrm{E}-09$ \\
\hline Transport of the SLBP Dependant Mature mRNA & R-HSA-159230 & 8 & 43.95 & $5.26 \mathrm{E}-11$ & 4.45E-09 \\
\hline SUMOylation of chromatin organization proteins & R-HSA-4551638 & 9 & 30.79 & $5.20 \mathrm{E}-11$ & $4.57 \mathrm{E}-09$ \\
\hline
\end{tabular}

J Proteome Res. Author manuscript; available in PMC 2021 November 09. 